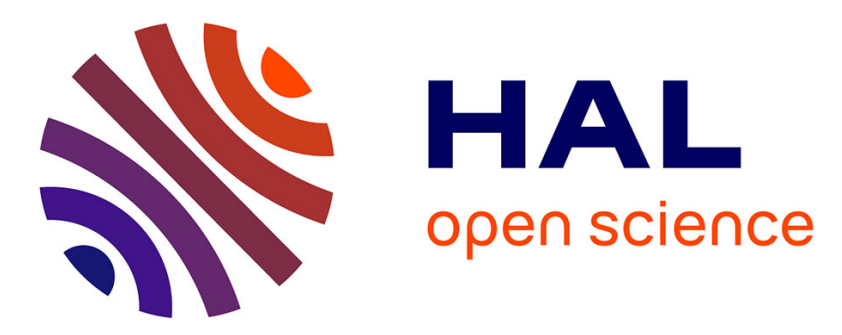

\title{
Une expérimentation de deux modes de scrutin en Martinique lors du premier tour de l'élection présidentielle de 2017
}

Eric Kamwa, Gilles Joseph, Aurélie Roger

\section{- To cite this version:}

Eric Kamwa, Gilles Joseph, Aurélie Roger. Une expérimentation de deux modes de scrutin en Martinique lors du premier tour de l'élection présidentielle de 2017. Revue d'Economie Politique, 2020, 130 (5), pp.759-798. hal-01965660v2

\section{HAL Id: hal-01965660 \\ https://hal.univ-antilles.fr/hal-01965660v2}

Submitted on 9 Nov 2020

HAL is a multi-disciplinary open access archive for the deposit and dissemination of scientific research documents, whether they are published or not. The documents may come from teaching and research institutions in France or abroad, or from public or private research centers.
L'archive ouverte pluridisciplinaire HAL, est destinée au dépôt et à la diffusion de documents scientifiques de niveau recherche, publiés ou non, émanant des établissements d'enseignement et de recherche français ou étrangers, des laboratoires publics ou privés. 


\title{
Une expérimentation de deux modes de scrutin en Martinique lors du premier tour de l'élection présidentielle de $2017^{*}$
}

\author{
Eric Kamwa ${ }^{\dagger}$
}

\author{
Gilles Joseph
}

Aurélie Roger $\$$

7 avril 2020

\begin{abstract}
Résumé
Lors du premier tour de l'élection présidentielle du 22 avril 2017, les électeurs de trois bureaux de vote de la commune de Fort-de-France (Martinique) ont été sollicités en vue de tester deux modes de vote : le Vote Alternatif et la règle de Borda à 4. L'objectif de ce papier est de rendre compte des résultats de cette expérimentation qui est la première du genre dans les territoires d'outre-mer. Les résultats obtenus font apparaitre des divergences avec les résultats officiels tant au niveau local que national. Sur la base des résultats bruts, en plus d'être le vainqueur sous chacune des règles testées, J.-L. Mélenchon est également le vainqueur de Condorcet alors que J. Cheminade est le perdant de Condorcet sous le vote Alternatif et M. Le Pen est le perdant de Condorcet sous la règle de Borda à 4 . Cependant, après correction du biais de représentativité et de participation, E. Macron devient le vainqueur pour chacune des règles testées.
\end{abstract}

Classification JEL : C93, D71, D72.

Mots-clés : Vote Alternatif ; Règle de Borda ; Expérimentation; Classements ; Condorcet.

${ }^{*}$ Les auteurs souhaitent remercier le services des élections de la préfecture de la Martinique, Madame Mylène Confiant du service des élections de la mairie de Fort-de-France. Nos remerciements vont également à Vincent Merlin et Isabelle Lebon qui nous ont assisté dans la conception des supports de l'expérimentation, Marcellus Harper pour son aide dans la conception de la feuille de calculs utilisée pour le vote alternatif. Enfin, cette expérimentation n'aurait pas été possible sans le concours des étudiants et collègues qui nous ont aidé et assisté tout au long de l'expérimentation, en particulier dans la tenue du bureau de vote, nécessitant force de conviction pour convaincre les électeurs de participer et efforts pédagogiques constants pour leur expliquer les règles de vote, pendant toute la journée du scrutin : Myriem Aqel, Tristan Damba, Aurélie Eliazord, Charles Haranis, Philippe Simon et Ibrahima Dia, que nous remercions également. Pour finir, nous n'oublierons pas Jean-François Laslier et ainsi que trois rapporteurs anonymes pour leurs remarques pertinentes qui ont permis d'améliorer la première version de ce papier.

${ }^{\dagger}$ Corresponding author. LC2S UMR CNRS 8053, Univ. des Antilles, Faculté de Droit et d'Economie de la Martinique, F97209 Schoelcher, Martinique. (eric.kamwa@univ-antilles.fr).

${ }^{\ddagger}$ LC2S UMR CNRS 8053, Univ., Faculté de Droit et d’Economie de la Martinique, F97209 Schoelcher, Martinique. (gilles.joseph@univ-antilles.fr)

${ }^{\S}$ LC2S UMR CNRS 8053, Univ. des Antilles, Faculté de Droit et d'Economie de la Martinique, F97209 Schoelcher, Martinique. (aurelie.roger@univ-antilles.fr) 


\title{
An Experiment of two Voting Methods in Martinique during the first round of the 2017 Presidential Election
}

\begin{abstract}
During the first round of the presidential election on April 22, 2017, voters from three polling stations in the municipality of Fort-de-France (Martinique) were asked to test two voting methods : the Alternative Vote and the Borda rule at 4. The purpose of this paper is to report the results of this experiment which is the first of its kind in the overseas territories. The results obtained show discrepancies with official results at both local and national levels. On the basis of the gross results, in addition to being the winner under each of the rules tested, J.-L. Mélenchon is also the Condorcet winner while J. Cheminade is the Condorcet loser under the alternative vote and M. Le Pen is the Condorcet loser under the rule of Borda at 4 . After correcting the bias of representativeness and participation, E. Macron becomes the winner for each rule tested.
\end{abstract}

JEL Classification Number : C93, D71, D72.

Keywords : Alternative Vote; Borda Rule; Experiment; Rankings; Condorcet.

\section{Introduction}

L'élection présidentielle constitue un moment central dans la vie démocratique d'un certain nombre nombre de pays, tels les Etats-Unis ou la France, où le pouvoir exécutif est détenu de manière effective seul ou conjointement par un président qu'elle a pour fonction de désigner. D'un pays à l'autre, l'élection du président est conduite sous l'un des deux principaux modes de suffrage suivants : i) le suffrage indirect dans lequel les électeurs ne choisissent pas eux-mêmes la personne qu'ils souhaitent voir élue, mais élisent des personnes qui font ce choix. Dans ce système, le président peut être, par exemple, élu par le Parlement, ou par des grands électeurs qui n'ont pas d'autres fonctions, comme aux Etats-Unis. ii) le suffrage direct où le vote de chaque électeur est comptabilisé pour désigner le vainqueur de l'élection. Depuis le référendum de 1962 et le scrutin de 1965, l'élection du président en France s'effectue au suffrage universel direct. Les modalités de déroulement du scrutin sont fixées par les articles 6,7 et 58 de la Constitution française et par la loi organique du 6 novembre 1962. La règle de vote utilisée est le scrutin majoritaire uninominal à deux tours : au premier tour, l'électeur vote pour un candidat parmi tous les candidats en lice. Si un candidat recueille la majorité absolue (plus de 50\% des suffrages exprimés), il est élu, sinon, un second tour est organisé entre les deux candidats arrivés en tête lors du premier tour, et le candidat élu est celui qui recueille la majorité absolue des voix.

Tout mode de scrutin, en tant que règle de vote permettant l'agrégation d'opinions individuelles pour produire un choix collectif, mérite d'être interrogé du point de vue de ses propriétés, vertus et limites. De nombreux travaux ont été développés dans ce sens. Il ressort des célèbres théorèmes d'Arrow (1951) et de Gibbard-Satterthwaite (Gibbard, 1973, Satterthwaite, 1975) qu'il n'existe aucun système de vote idéal. Le scrutin majoritaire uninominal à deux tours a l'avantage d'être caractérisé par une forme de simplicité dans la production du résultat électoral. Pour Sato (2016), la raison principale pour laquelle cette règle (tout 
comme la règle de la Pluralité) est largement utilisée dans les sociétés réelles est qu'elle possède la propriété d'efficacité de l'information, qui est une caractéristique pratiquement importante des règles de vote. Le scrutin majoritaire uninominal à deux tours reste toutefois sujet à certaines critiques tant sur le plan théorique que pratique. Sur le plan théorique, la propension de cette règle à conduire à des paradoxes de vote ${ }^{1}$ n'est pas négligeable. Le lecteur peut à ce propos consulter les travaux de Lepelley et Merlin (1999), Saari (1994, 2003). Sur le plan pratique, il lui est classiquement reproché de favoriser la dispersion des voix des électeurs lors du premier tour, mais aussi l'abstention ou d'autres formes de défiances à l'égard du jeu politique chez les électeurs des partis dont il défavorise l'accès au pouvoir ${ }^{2}$ par opposition au scrutin proportionnel (Norris, 1997, Gallagher et Mitchell, 2005). On peut également noter que lors des élections présidentielles françaises de 2007, 2012 ou 2017, le scrutin uninominal à deux tours a incité les électeurs à voter stratégiquement ou utile en se concentrant sur peu de candidats (Baujard et Igersheim, 2007).

Tout en gardant à l'esprit qu'il n'existe aucun mode de suffrage idéal, il est intéressant dès lors de considérer des modes d'élection alternatifs au scrutin uninominal à deux tours et d'observer leurs vertus comparatives. L'actualité française y incite, notamment à travers les débats récurrents autour de l'introduction du suffrage proportionnel pour les élections législatives. Remise à l'ordre du jour par le Président Macron lors de sa campagne présidentielle, à travers la proposition d'un scrutin mixte permettant d'élire une part minoritaire de députés via ce mode de scrutin. La question a été réactivée durant l'hiver 2018-2019 par le mouvement des gilets jaunes dont l'aspiration à une meilleure représentativité tant politique que sociale des élus s'est traduite par la revendication fréquente d'une proportionnelle intégrale - du moins lorsque le principe d'une désignation par l'élection était accepté, puisque la question du tirage au sort a joui elle aussi d'une forte popularité parmi les groupes mobilisés. Cette actualité récente montre combien la question du mode de désignation des représentants politiques et du degré de confiance et de satisfaction des citoyens à son égard n'a rien de purement théorique mais renvoie à des enjeux démocratiques qui sont au cœur des préoccupations sociales contemporaines. Le débat sur les effets des modes de scrutin et sur le degré de conformité de leurs résultats aux aspirations des citoyens n'en est que plus central.

Norris (1997), à la suite d'une longue tradition d'analyse de l'influence, réelle ou supposée, des modes de scrutin sur les systèmes politiques (Duverger, 1950, Martin, 1996), fait remarquer qu'une réforme électorale n'est jamais anodine car, selon la société dans laquelle elle est mise en œuvre, elle peut conduire à des systèmes politiques instables, déboucher sur une forte polarisation politique, donner un rôle de "faiseurs" de rois à certains groupes (à l'exemple des minorités) ou alors favoriser certains type de candidats (Baujard et al., 2014). Cette tradition d'analyse est toutefois largement cantonnée aux vertus et limites comparées des scrutins majoritaire et proportionnel, de leurs déclinaisons mixtes, et du nombre de tours de scrutins. Elle explore assez peu, en revanche, des modalités de vote plus originales, qui font pourtant pour certaines l'objet d'applications éparses, après des réflexions intenses au

1. Un paradoxe de vote est un résultat contre-intuitif qui peut résulter de l'agrégation des préférences individuelles. Pour un exposé sur les paradoxes de vote, le lecteur peut consulter les livres de Nurmi (1999) et Felsenthal et Nurmi (2018).

2. Il s'agit notamment des petits partis éliminés au premier tour, partis incapables de conclure des alliances en vue du second tour. 
siècle des Lumières, notamment en France dans les années 1770 et 1780, autour des travaux de jeunes savants siégeant à l'Académie royale des sciences, tels Condorcet, Borda ou Laplace, passionnés par la rencontre féconde entre la mathématique des suffrages et la toute jeune science économique (Brian, 2001). D’après Norris (1997), toute réforme électorale doit répondre à des objectifs précis et donner lieu à des arbitrages francs tant sur ses objectifs que sur les critères normatifs que doit satisfaire la règle de vote à mettre en œuvre; cette règle de vote doit dans tous les cas être simple et compréhensible pour l'électeur. Le scrutin majoritaire uninominal à deux tours, bien installé dans le paysage politique français de la Ve République, tant pour l'élection présidentielle que pour les élections législatives, remplit incontestablement ces derniers critères. De sorte que l'introduction de nouvelles règles peut facilement, à rebours, être perçue comme complexifiant le scrutin pour l'électeur. Dans ces conditions, un possible préalable à une réforme électorale pourrait dès lors consister à soumettre une nouvelle règle de vote à l'appréciation des électeurs par le biais d'expérimentations au cours desquelles ils la testeraient et seraient informés sur ses impacts. Dans cette optique, une expérimentation de terrain a été menée lors du premier tour de l'élection présidentielle de 2002 au cours de laquelle le "vote par approbation" 3 a été testé à grande échelle (Balinski et Laraki, 2011, Balinski et al., 2003, Laslier et Van der Straeten, 2004, 2008). D'autres modes de scrutin ont été testés lors de l'élection de 2007 : le jugement majoritaire ${ }^{4}$ (Balinski et Laraki, 2011), le vote transférable ${ }^{5}$ (Farvaque et al., 2009, 2011), le vote par approbation et le vote par note ${ }^{6}$ (Baujard et Igersheim, 2007, 2009). Pour plus de résultats d'expérimentation de la règle du vote par note, le lecteur peut consulter les travaux de Baujard et al. (2011, 2018, 2013), Igersheim et al. (2016) ou encore le récent opuscule de Laslier (2019).

Une expérimentation électorale aide à mieux comprendre le fonctionnement des institutions démocratiques, à étudier les propriétés des procédures de décision collective ainsi que le comportement des électeurs face aux modes de scrutin (Igersheim et Baujard, 2019). L'appel d'offre lancé le 17 Octobre 2006 par le Centre d'Analyse Stratégique portant sur les "Etudes monographiques sur une ou plusieurs expérimentations engagées en matière d'organisation des consultations électorales nationales ou locales", témoigne de l'importance que

3. Le vote par approbation, permet à chaque électeur d'accorder son soutien à autant de candidats qu'il le souhaite. Un même électeur peut donc soutenir zéro, un, deux, voire tous les candidats. Est élu celui qui rassemble le plus grand nombre de soutiens.

4. Sous cette règle, les électeurs ne classent pas implicitement les candidats mais leur attribuent des appréciations. Le vainqueur est déterminé à partir de la médiane des appréciations.

5. Dans la version la plus répandue du vote transférable (utilisée par exemple depuis le tournant des années 1910 et 1920 en Australie et en Irlande pour l'élection de la chambre des représentants), le vote alternatif encore appelée méthode de Hare (1859) fonctionne de la manière suivante : le candidat ayant recueilli le plus faible nombre de premières places dans les classements des électeurs est éliminé et ses voix sont transférées aux candidats que chaque électeur a classé après lui. Si à la suite du transfert, un candidat obtient la majorité des voix, il est élu; dans le cas contraire, une nouvelle itération s'opère, et ainsi de suite jusqu'à l'émergence d'un vainqueur. Notons qu'à côté de cette version populaire du vote transférable, il existe la méthode de Coombs qui elle élimine les candidats sur la base du nombre de fois où ils sont classés en dernière position. Ainsi, à chaque itération, est éliminé, le candidat ayant le plus grand nombre de dernières places.

6. Le vote par note est un mode de scrutin consiste à attribuer une note à chaque candidat $(0,1$ ou 2$)$, le nombre de points réunis par chacun des candidats détermine le résultat de l'élection. Est élu celui qui obtient le total le plus élevé. 
les pouvoirs publics accordent aux expérimentations. La France n'est pas le seul pays où des expérimentations ont été réalisées lors de scrutins officiels. Des expériences similaires ont été menées en Allemagne (Alœs-Ferrer et Granic, 2010), au Bénin (Kabre et al., 2012). Des expériences du même genre, mais sur Internet, ont aussi été menées au Canada, en France et en Islande (Van der Straeten et al., 2013, Blais et al., 2015), et plus récemment en Autriche (Darmann et al., 2017). Le lecteur pourra se référer à la remarquable revue de la littérature faite par Baujard et al. (2016) pour avoir un aperçu des différentes expérimentations menées en France lors des élections présidentielles de 2012.

Lors de l'élection présidentielle française de 2017, plusieurs expérimentations in situ ont été menées à travers le pays : Allevard-les-Bains (Isère), Crolles (Isère), Grenoble (Isère), Hérouville Saint-Clair (Calvados), Strasbourg (Bas-Rhin) et Fort-de-France (Martinique). Si l'on considère l'ensemble des sites expérimentaux de l'Hexagone, les règles suivantes ont été testées : le vote par approbation, le vote par note et le vote par approbation à deux tours. Le lecteur peut consulter librement les résultats de ces expérimentations sur le site du GATE ${ }^{7}$. A côté de ces expérimentations in situ, il convient aussi de noter l'expérimentation menée sur internet via le site "Voter Autrement" (https://vote.imag.fr/). Ce site proposait de tester le vote par approbation, le vote par note (avec des échelles différentes), la règle de Borda à 4 et le vote par élimination successive. Les résultats de cette expérimentation sont disponibles sur le site dédié. Le nombre de participants aux différentes expérimentations témoigne bien de l'intérêt et de la curiosité des électeurs quant à l'usage d'une règle de vote alternative à celle en vigueur pour le scrutin officiel.

L'expérimentation que nous avons menée dans plusieurs bureaux de vote de la ville de Fort-de-France est la première à avoir été réalisée dans les territoires français d'outre-mer. Lors de cette expérimentation, il a été proposé aux électeurs de tester deux règles de vote : le Vote Alternatif et la règle de Borda à 4. Avec le Vote Alternatif, il est demandé aux électeurs de classer tous les candidats; une fois les décomptes faits, le candidat ayant été le moins de fois classé en tête (premier) est éliminé et ses voix sont transférées aux candidats que les électeurs concernés ont classés en deuxième position. Autrement dit, l'électeur qui avait classé le candidat éliminé en première position transfère son vote au second de sa liste. Si à la suite de tous les transferts, un candidat obtient la majorité des voix, il est élu; dans le cas contraire, une nouvelle itération s'opère, et ainsi de suite jusqu'à l'émergence d'un vainqueur. La règle de Borda à 4 qui est une version tronquée de la règle originelle de Borda (1781) exige des électeurs de classer 4 candidats dans un ordre strict du plus préféré au moins préféré; chaque fois qu'un candidat est classé premier il reçoit 4 points, deuxième il reçoit 3 points, troisième il est crédité de 2 points, il reçoit 1 point lorsqu'il est classé quatrième et aucun point lorsqu'il n'est pas classé. Le vainqueur est le candidat ayant récolté le plus grand nombre de points.

Il est vrai que le vote alternatif est réputé pour sa complexité de par l'information demandée aux électeurs (classement complet) et sa méthode de dépouillement. D'après Hare (1859), cette règle offre à l'électeur l'opportunité d'exprimer toute forme d'opinion politique ; sur le plan normatif, cette règle, semble posséder de meilleures propriétés et à la complexité qui lui est associée se greffe une faible vulnérabilité à des paradoxes de votes (Felsenthal et Nurmi, 2018, Grofman et Feld, 2004). Pour plus d'arguments en faveur du vote alternatif,

7. https://www.gate.cnrs.fr/IMG/pdf/_2019-01-18_cr_global_v2.pdf. 
le lecteur pourra se référer aux travaux de Farvaque et al. (2009, 2011), Grofman et Feld (2004), Farrell et al. (2000), Reilly et Maley (2000). Notons également que nous avons opté pour le vote alternatif dans un souci de complémentarité avec les autres expérimentations menées en 2017. Sur le plan normatif, la littérature du choix social classe la règle de Borda et la règle de la Pluralité (qui gouverne le premier tour de l'élection présidentielle) dans la famille des "règles de scores". Dans cette famille, la règle de Borda tend à se distinguer de toutes les autres de par ses propriétés et une meilleure résistance à divers paradoxes de vote et comportements stratégiques. A ce propos, voir les travaux de Saari $(1994,2003)$. De plus, la règle de Borda a tendance à favoriser le(s) candidat(s) soutenu(s) par un large consensus parmi les électeurs plutôt que le candidat qui est nécessairement le favori d'une majorité. C'est donc fort de cet argument que nous avons opté pour la règle de Borda. Ici, le recours à la version tronquée (Borda à 4) rejoint l'idée de réduire la complexité liée à l'information demandée et également le souci de complémentarité avec les autres expérimentations menées en 2017.

L'expérimentation électorale ainsi menée a pour objectif d'observer l'incidence de ces deux règles sur le comportement des électeurs. Il peut en effet sembler être d'autant plus compliqué, et donc rebutant, pour un électeur de classer tous les candidats, que ces derniers sont nombreux, ce qui est souvent le cas au premier tour de l'élection présidentielle française. Par cette expérimentation nous avons voulu appréhender cette complexité et vérifier la cohérence des choix des électeurs entre les deux règles de vote testées. Il s'agit d'autre part d'appréhender l'influence que peuvent avoir ces règles sur la traduction en résultat électoral des préférences entre candidats exprimées par les électeurs à travers leur vote. La comparaison avec les autres expérimentations menées ailleurs en France en 2017 ou lors de scrutins présidentiels antérieurs permet d'une part de mettre les hypothèses élaborées dans le cadre de ces travaux à l'épreuve d'un nouveau terrain d'enquête présentant certaines singularités électorales fortes. D'autre part, elle autorise l'observation des effets de la conjoncture politique sur les résultats produits, compte tenu de l'originalité de l'offre électorale proposée aux citoyens français en 2017, au regard, par exemple, de celle de 2007 où l'expérimentation du vote alternatif avait également pu être menée (Farvaque et al., 2009).

Le reste de l'article s'organise de la manière suivante : La Section 2 présente brièvement la scène politique martiniquaise, qui se démarque du contexte politique hexagonal par certaines spécificités. Dans la Section 3, nous exposons le déroulement de l'expérimentation et résumons les principales caractéristiques des participants. Les résultats de l'expérimentation sont donnés dans la Section 4 et la Section 5 conclut.

\section{Vie politique martiniquaise et vote à l'élection prési- dentielle}

Mener une expérimentation électorale en Martinique, et plus singulièrement encore à Fort-de-France, n'est pas immédiatement comparable avec d'autres terrains investis par les chercheurs au cours du même scrutin. La thèse de l'homogénéisation spatiale des comportements électoraux à l'échelle nationale française (Dolez et Laurent, 2001) est certes apparue remise en cause ces dernières années, notamment par la répartition géographique spécifique du vote pour le Front national à l'échelle de l'Hexagone (Gombin, 2015, Huc, 2019). Mais le 
vote des outre-mer, et en particulier des Antilles, s'est lui singularisé de longue date du vote national, au point de résister dès l'abord à cette hypothèse d'une homogénéisation.

L'élection présidentielle constitue en premier lieu, pour les électeurs martiniquais, une occasion de se positionner sur une offre politique différente de celle qui leur est proposée dans le cadre de la plupart des autres scrutins auxquels ils sont conviés. La vie politique martiniquaise se structure en effet autour d'un paysage partisan et d'un clivage politique local distincts de ceux qui organisent le débat national. Rapidement déçues par les promesses égalitaires non tenues de la départementalisation de cette ancienne colonie en 1946, les forces politiques de gauche se sont, à partir des années 1950, affirmées dans une double rupture : rupture organisationnelle avec les partis nationaux, d'une part, dont l'intégration était jugée inapte à assurer le respect des spécificités locales, générant la création de partis locaux sans lien d'intégration avec les grandes fédérations partisanes nationales; rupture idéologique avec le bilan politique de la départementalisation, ensuite, face à la persistance de problèmes économiques et sociaux non résolus, d'inégalités devant les lois sociales et de répressions violentes des mouvements sociaux, générant des projets politiques d'évolution institutionnelle de l'île, sous la forme d'une émancipation à l'égard de la tutelle de l'Etat français.

C'est ainsi qu'à gauche, depuis les années 1980, les élections locales voient s'affronter deux forces politiques principales, attirant dans leur mouvance un certain nombre de partis de moindre envergure avec lesquels ils forment des coalitions : le Parti progressiste martiniquais (PPM), d'une part, fondé par Aimé Césaire en 1958, tenant d'un projet autonomiste, et le Mouvement indépendantiste martiniquais (MIM), d'autre part, fondé en 1978 autour d'un projet initialement indépendantiste. Par contraste, les forces de droite ont longtemps maintenu un lien d'affiliation aux partis nationaux (aujourd'hui Les Républicains) et revendiqué à l'égard de l'Etat une ligne pleinement assimilationniste. Des partis locaux ont cependant là aussi été créés à partir des années 1990 et certains d'entre eux ont pu s'ouvrir à la question de l'évolution statutaire. En tout état de cause, la structuration du débat politique qui vient d'être décrite supplante largement les autres formes d'opposition ayant cours au niveau national et irrigue jusqu'à aujourd'hui les représentations du clivage droite/gauche chez les électeurs martiniquais.

Le débat politique national, évidemment, n'est pas pour autant étranger aux électeurs martiniquais, les médias nationaux et locaux s'en faisant l'écho. Par ailleurs, les forces politiques locales, même lorsqu'elles n'appartiennent pas aux partis nationaux, développent avec eux des liens de proximité entretenus par le jeu des affiliations aux groupes parlementaires à l'Assemblée nationale et au Sénat. Les parlementaires du PPM siègent ainsi traditionnellement au sein du groupe rassemblant les élus socialistes, et ceux du MIM aux côtés des communistes.

La campagne présidentielle est dans ces conditions relayée par les forces locales en fonction de ces liens, avec des effets visibles en termes de mobilisation électorale. Le PPM, qui constitue depuis sa création la force politique dominante à Fort-de-France, fait ainsi traditionnellement campagne en faveur du candidat socialiste à la présidence de la République. En 2017, 13, 24\% des électeurs de cette commune ont ainsi porté leur scrutin sur Benoît Hamon, donnant à ce candidat, qui a recueilli $9,76 \%$ des voix en Martinique et $6,36 \%$ au niveau national, son second meilleur score de l'île, immédiatement derrière une commune détenue par un maire socialiste. Si l'écart de voix est parlant, il est cependant hors de proportion avec les résultats traditionnels des candidats socialistes dans la commune, et lié au 
contexte politique national de faible soutien des élus socialistes à la candidature officielle, ainsi qu'aux conséquences de la primaire, qui avait vu les forces politiques locales et les électeurs de gauche soutenir massivement la candidature de Manuel Valls.

De façon générale, on notera que la domination de la vie politique locale, au niveau du territoire, par les forces politiques de gauche, se retrouve de manière patente dans les résultats des élections présidentielles. Les Martiniquais ont pourtant longtemps voté à ces élections pour les candidats de droite (en particulier gaullistes), y compris de façon massive lors de l'élection de 1981, où F. Mitterrand l'emportait au niveau national. Mais une fois levée la crainte du "largage" par la France, autonomie ou indépendance accordée de force par l'Etat dont le spectre était associé dans l'esprit des électeurs aux gouvernants de la gauche nationale, ils ont depuis 1988 durablement et fortement voté pour les candidats de gauche aux premier et second tours de chaque scrutin présidentiel. F. Hollande a par exemple obtenu près de $52 \%$ des voix dès le premier tour en 2012. L'autre singularité notable que présente le vote à l'élection présidentielle en Martinique, quoique commune à l'ensemble des territoires d'outre-mer, est le très fort taux d'abstention qui s'y manifeste. Effet sans doute de la perception lointaine des enjeux nationaux, ce taux est de deux à trois fois supérieur à la moyenne nationale suivant les années. En 2017, 60,12\% des électeurs martiniquais ne se sont pas rendus aux urnes au premier tour de l'élection présidentielle (contre 22, 23\% au niveau national). Un autre aspect remarquable est que cette abstention est systématiquement en recul net au second tour $(-10,38 \%$ en 2017$)$, ce qui laisse penser que la réduction du choix opérée entre les deux tours a, de manière contraire à ce qui se produit au niveau national où une partie des électeurs des partis éliminés ont tendance à se désintéresser du scrutin entre les deux tours, plutôt pour effet de remobiliser l'électorat martiniquais.

L'expérimentation électorale qui a été menée s'inscrit donc dans ce contexte singulier, au regard duquel ses résultats méritent d'être analysés.

\section{Le déroulement de l'expérimentation et les caractéris- tiques des électeurs}

\subsection{Le déroulement de l'expérimentation}

Après les autorisations de la préfecture, nous avons bénéficié pour la mise en œuvre de l'expérimentation, du soutien du service de communication et de la logistique de la Mairie de Fort-de-France. Les nombreux échanges avec le service des élections de la Mairie nous ont conduits à mener l'expérimentation à l'Ecole Dillon D qui abritait les bureaux 17, 18 et 19 de la ville de Fort-de-France. Ce choix a été fait sur la base des statistiques de participation dans les différents bureaux de vote lors des derniers scrutins. Il s'agissait pour nous d'avoir accès à une population d'électeurs suffisante pour que l'expérimentation porte sur un nombre de votes significatif. Or, les taux d'abstention sont particulièrement élevés dans l'ensemble de la Martinique et particulièrement dans les 60 bureaux de Fort-de-France, et nous n'avions pas d'assurance quant à l'adhésion des électeurs à l'expérimentation. Le choix a donc été fait de nous installer dans le lieu de vote qui permettrait d'optimiser nos chances de recueillir des votes expérimentaux. D'après les données mises à dispositions par la Mairie de Fort de France, l'Ecole Dillon D, présentant l'intérêt de rassembler 3 bureaux, était en outre un site 
de vote où les taux de participation étaient traditionnellement parmi les plus élevés de la ville. C'est ainsi qu'elle a été choisie. On notera cependant que ces caractéristiques en font, dès l'abord, un lieu de vote présentant quelques spécificités à l'échelle de la Martinique, dont certaines incidences seront discutées plus loin.

Les électeurs de l'Ecole Dillon D ont été informés de l'expérimentation par divers moyens (affiches, annonces, canaux de communication de la Mairie) ${ }^{8}$ et par une intervention sur les antennes d'une des radios les plus écoutées de l'île. Une page internet a été conçue et tout électeur pouvait avant l'expérimentation se familiariser avec les règles de vote devant être testées ${ }^{9}$.

Le samedi ${ }^{10} 22$ Avril 2017, date du premier tour, le bureau expérimental a ouvert et fermé aux mêmes heures que les bureaux officiels, dans une salle de classe distincte située juste en face de celles qui abritaient les bureaux officiels. Les électeurs se voyaient indiquer dès leur arrivée au portail de l'école - au niveau duquel des affiches explicatives avaient été apposées- la possibilité de tester d'autres règles de vote et étaient invités sur la base du volontariat à participer à l'expérimentation une fois leur devoir citoyen accompli. Une fois dans le bureau expérimental, l'électeur se voyait expliquer oralement les deux règles de vote proposées, sous la forme d'une présentation de la manière de voter et de la manière d'obtenir les résultats à partir des votes. Chacun d'entre eux recevait un bulletin (voir un exemple en Annexe) sur lequel étaient rappelées de manière écrite les règles de vote. La salle étant grande, cependant, le choix a été opéré de mettre à disposition plusieurs tables et chaises en des points éloignés, qui permettaient aux électeurs de voter en s'isolant des regards. Deux urnes opaques leur étaient offertes pour y déposer eux-mêmes leur bulletin, une fois rempli.

Ce dispositif a donné lieu à des usages divers. Un certain nombre d'électeurs ont interpellé en cours de vote les membres de l'équipe pour se faire rappeler l'une ou l'autre règle, voire parfois se faire aider à remplir leur bulletin. D'autres, venus voter en famille, ont été observés s'aidant mutuellement. On perçoit donc là une difficulté liée à l'introduction d'une règle de vote non maîtrisée, rappelant les descriptions socio-historiques de premiers scrutins au suffrage universel - et les arguments utilisés par la suite par les contempteurs de l'introduction de l'isoloir, qui défendaient l'impérieuse nécessité pour certains électeurs de se faire aider dans l'expression de leur suffrage (Garrigou, 1988). Les expérimentateurs se sont évidemment attachés de leur côté à ne jamais influer sur l'expression des préférences opérée à travers les classements et à se cantonner à un rôle d'explicitation de la règle. On peut relativiser l'importance de ces faits en considérant qu'ils sont largement liés à une exposition insuffisante de ces électeurs, en amont de l'expérimentation, à la présentation pédagogique de la règle de vote, qui ne manquerait pas de se produire de manière appuyée et récurrente en cas

8. Il n'y a pas eu d'envoi de lettres au électeurs comme cela est le cas dans la plupart des expérimentations. Ce choix est le fruit des discussions avec les services des élections de la Mairie en se basant sur la spécificité et la particularité du terrain exploré.

9. Notons que ce sont près de 200 personnes réparties sur toute la Martinique qui ont passé le test sur cette page internet.

10. Depuis 2007, l'élection se tient le samedi du fait du décalage horaire avec l'Hexagone, où elle a lieu le dimanche. Cette réforme a mis fin à la situation antérieure, perçue comme démobilisatrice, où les électeurs se voyaient annoncer le résultat national de l'élection plusieurs heures avant la clôture de leur propre bureau de vote, au risque de leur donner le sentiment d'un vote sans aucun impact. La réforme n'a pas entraîné sur le long terme la diminution de l'abstention escomptée cependant, comme en témoigne le taux historiquement élevé relevé au 1er tour en 2017. 
de changement officiel du mode de scrutin. Mais on ne peut exclure pour autant qu'un tel changement n'aurait pas d'effets d'autocensure et d'auto-exclusion sur les électeurs les plus sensibles aux mécanismes d'inhibition liés au cens culturel caché déjà associés à l'exercice de la citoyenneté dans le cadre de la règle de vote officielle (Gaxie, 1978). Quoi qu'il en soit, l'absence d'isoloir et les interactions qu'elle a permises avec les membres de l'équipe au sujet de l'explicitation de la règle de vote ont sans doute favorisé le très bon taux de bulletins valides enregistré, qui sera évoqué plus loin.

\subsection{Caractéristiques des électeurs}

D'après les chiffres officiels ${ }^{11}$, sur les 5217 électeurs inscrits pour l'ensemble des bureaux de Dillon D, seuls 2217 se sont rendus aux urnes (soit 42, 49\% des inscrits). Parmi ces électeurs, 524 ont accepté de prendre part à l'expérimentation, soit un taux de participation de 23,64\%. Ce niveau de participation est inférieur à ceux observés dans l'ensemble des autres expérimentations menées à l'occasion des élections présidentielles françaises. Celui-ci est cela dit variable d'un cas à l'autre : il oscillait entre 54 et $78 \%$ lors de celles de 2002, 2007 et 2012 (Igersheim et al., 2016) et a même pu descendre jusqu'à 36\% dans une commune lors de l'expérimentation "Voter autrement" ${ }^{12}$ en 2017 . On peut voir malgré tout dans la plus faible participation que nous avons enregistrée un effet de l'insuffisante information préalable des électeurs, faute de diffusion individuelle par la mairie de la lettre préparée à leur intention. Les autres canaux d'information mobilisés n'ont sans doute pas touché tous les électeurs en amont de leur arrivée dans le bureau, et la découverte "à chaud" de l'expérimentation ne doit pas avoir facilité la mobilisation des électeurs. De fait, un certain nombre de personnes ont décliné dès leur accueil à l'entrée de la cour de l'école. Il s'agit donc là d'un premier facteur explicatif. Il convient aussi de noter que contrairement à ce qui a pu se passer dans d'autres expérimentations, une salle dans un bâtiment distinct du vote officiel - bâtiment séparé par une portion de la cour menant à la sortie de l'école, même s'il n'était éloigné que d'une dizaine de mètres - a été attribuée par la mairie, localisation elle aussi susceptible de générer une déperdition. Il serait intéressant de ce point de vue de pouvoir opérer, entre les différentes expérimentations qui ont fait l'objet de publications, des comparaisons des taux de participation selon les configurations matérielles du vote expérimental, son éloignement du vote officiel et le passage plus ou moins contraint devant sa porte. En l'état, les données ne sont pas disponibles dans ces travaux pour nous assurer qu'il s'agit d'un facteur déterminant. Afin de limiter l'influence de ces deux facteurs, le choix avait été opéré, comme nous l'avons signalé, d'évoquer l'expérimentation auprès des électeurs dès leur passage du portail de l'école, en les invitant à y participer après leur vote officiel. Les expérimentateurs ainsi positionnés s'assuraient également auprès des électeurs quittant l'école de leur bonne participation au dispositif. Cette configuration impose un groupe fourni d'expérimentateurs afin de pouvoir assurer cette mission en même temps que celle d'accompagnement des électeurs dans le vote à l'intérieur du bureau. Compte tenu de diverses difficultés et défections, neuf volontaires ont en l'espèce rempli l'ensemble de ces fonctions conjointement. Lors des périodes de plus grosse affluence, certains électeurs ont cependant échappé à leur action.

11. Sources : Services des élections de la Mairie de Fort-De-France.

12. Voir le dernier rapport des expérimentations, https://www.gate.cnrs.fr/IMG/pdf/_2019-01-18_ cr_global_v2.pdf. 
Enfin, on notera qu'après avoir essuyé un de ces épisodes d'arrivée massive, le réapprovisionnement en bulletins de la mi-journée a finalement été précédé d'une rupture des stocks pendant une trentaine de minutes - lors d'une période plus creuse heureusement. Quelques participants (une vingtaine) ont également été perdus à cette occasion. Il est intéressant d'observer qu'une quinzaine d'entre eux sont restés malgré tout dans le bureau en attendant les bulletins, témoignant par-là de leur fort intérêt pour le dispositif par-delà ce désagrément matériel.

Sur le bulletin de l'expérimentation, les électeurs devaient répondre à un bref questionnaire portant notamment sur le genre, la catégorie socio-professionnelle et la classe d'âge. Après un tri des questionnaires, 16 (soit 3\% des participants) n'étaient pas exploitables (questionnaires blancs ou mal remplis). Nous ne disposons malheureusement pas de données socio-démographiques concernant spécifiquement l'électorat des bureaux où s'est tenue l'expérimentation, ni a fortiori concernant les électeurs du 22 avril 2017, qui nous permettrait d'évaluer la représentativité des (électeurs) participants ou la surreprésentation de certaines catégories parmi ceux qui ont joué le jeu de l'expérimentation.

Parmi les 508 bulletins exploitables, 319 (62,79\%) provenaient d'électeurs féminins et 165 $(32,48 \%)$ d'électeurs masculins, 24 (4,72\%) n'ont pas indiqué leur sexe. La Table 1 permet d'avoir un aperçu des autres caractéristiques.

TABLE 1 - Répartition des des électeurs en fonction de l'âge et de la CSP

\begin{tabular}{|c|c|c|c|c|c|c|c|c|c|c|}
\hline & & \multicolumn{9}{|c|}{ Profession } \\
\hline & & Retraités & $\begin{array}{l}\text { Etudiants } \\
\text { Elèves }\end{array}$ & Chômeurs & $\begin{array}{l}\text { Artisans } \\
\text { Commerçants }\end{array}$ & Employés & Cadres & $\begin{array}{l}\text { Professions } \\
\text { intermédiaires }\end{array}$ & $\begin{array}{l}\text { Pas de } \\
\text { réponse }\end{array}$ & Total \\
\hline Âge & $18-29$ ans & 0 & 42 & 17 & 2 & 14 & 5 & 8 & 4 & 92 \\
\hline & $40-49$ ans & 0 & 0 & 24 & 12 & 24 & 18 & 27 & 4 & 109 \\
\hline & $50-59$ ans & 1 & 0 & 30 & 6 & 15 & 20 & 27 & 3 & 102 \\
\hline & $60-69$ ans & 22 & 0 & 21 & 3 & 7 & 5 & 9 & 6 & 73 \\
\hline & 70 ans et + & 21 & 0 & 14 & 1 & 0 & 0 & 0 & 3 & 39 \\
\hline
\end{tabular}

La catégorie socio-professionnelle la plus représentée est celle des chômeurs $(26,62 \%)$. Les tranches d'âge ayant le plus participé à l'expérimentation sont celles des 40-49 ans et des 50-59 ans. En l'absence de données sociologiques sur les électeurs qui ont voté à Dillon D ce jour-là, il ne nous est malheureusement pas possible de savoir si cette répartition est représentative de l'électorat du bureau, ce qui constitue une lacune de ces dispositifs expérimentaux sur laquelle nous reviendrons.

Nous pouvons à présent nous pencher sur les résultats de l'expérimentation.

\section{Les résultats des bureaux de l'Ecole Dillon D}

\subsection{Comment a-t-on voté à Dillon?}

Sur le questionnaire expérimental, les électeurs pouvaient indiquer leur choix lors du scrutin officiel. Parmi les 508 bulletins valides, 497 (soit 97,83\%) ont indiqué leur vote au 
scrutin officiel. Parmi eux, 47 avaient voté blanc, ce qui représente 23, $73 \%$ des 198 votes blancs recensés dans les bureaux concernés. Il est donc intéressant d'observer que des électeurs réputés n'avoir pas "exprimé" leur vote dans le scrutin officiel se montrent pour autant intéressés par le fait d'opérer un choix lorsqu'il peut être formulé de manière différente. Parmi eux, 46 (soit 97,87\%) ont opéré un vote valide avec la règle de vote alternatif, et 32 (soit 68,08\%) avec la règle de Borda à 4. Même s'il doit être pris avec précaution dans ce cadre expérimental, ce constat tend à accréditer l'idée selon laquelle, pour ce scrutin présidentiel, un vote plurinominal favoriserait davantage l'expression politique que le traditionnel scrutin uninominal à deux tours. Il ouvre également des interrogations sur l'effet remobilisateur que pourrait avoir une réforme électorale de cet ordre sur des franges de l'électorat de plus en plus portées à faire le choix de l'abstention, que l'expérimentation in situ n'a évidemment pas permis d'approcher.

L'analyse des votes blancs elle-même n'a jamais rien d'univoque, et les études montrent qu'ils tendent à agréger des comportements pluriels, de l'électeur "désinvesti" politiquement à celui qui souhaite au contraire exprimer une insatisfaction politique à l'égard de l'offre soumise à son suffrage, ou à faire entendre un véritable choix de ne pas choisir (Moualek, 2017). En l'occurrence, les chiffres évoqués mènent à formuler l'hypothèse que les électeurs des bureaux de Dillon qui ont accepté de participer à notre expérimentation appartiennent à la catégorie des votants officiels "blanc" la plus intéressée, la moins distante à l'égard de l'acte électoral, ou encore la plus "compétente". A Dillon D, par ailleurs, on peut postuler que se manifeste pour une part un ressort du vote blanc initialement mis en évidence en tant que caractéristique de la ruralité, puis étendu à tout contexte d'interconnaissance étroite : celui de substitut à l'abstention lorsque celle-ci n'est pas perçue comme socialement acceptable (Ranger, 1970). Dans le quartier de Dillon, certes urbain mais caractérisé par d'importants liens sociaux et par un fort encadrement partisan du PPM, les votes blancs et nuls pourraient bien avoir cette dimension particulière. On peut penser qu'un certain nombre d'électeurs ont réalisé un acte électoral contraint, sur fond de fort contrôle social imposant de ne pas être identifié comme abstentionniste sous peine de perdre les bénéfices associés au soutien du parti, qui depuis sa création en 1958 dirige la municipalité de Fort-de-France, mais que leur vote s'est pour autant traduit par une absence de choix entre les candidats, dans le contexte complexe de l'élection présidentielle de 2017 évoqué plus haut (soutien du PPM et des électeurs de gauche à M. Valls à la primaire, défections dans le camp socialiste au profit du candidat Macron au niveau national, tendance au vote de gauche "utile" en direction de J.-L. Mélenchon au fur et à mesure de la baisse du candidat Hamon dans les sondages). Les règles de vote proposées dans le cadre de l'expérimentation, en ce sens, pourraient aussi avoir été utilisées par ces électeurs, au contraire de la règle officielle, parce qu'elles leur offraient un cadre d" "expression" plus propice dans ce contexte particulier ${ }^{13}$. Au-delà du seul vote blanc, cette dernière hypothèse pourrait aussi expliquer le fait que $11 \%$ des électeurs ont classé un candidat en tête sous les règles expérimentales différent de celui pour lequel ils ont déclaré avoir voté officiellement. Ce chiffre est inférieur aux estimations de $22 \%$ issues de la

13. Pour le vérifier, il nous apparaît intéressant que ces expérimentations telles qu'elles se déroulent actuellement, selon des protocoles relativement éprouvés, puissent se doubler d'un volet d'enquête de nature qualitative, où des entretiens avec les électeurs participant à l'expérimentation permettraient de recueillir leurs motivations. Une telle méthode nécessite évidemment un dispositif plus lourd et la mobilisation d'un groupe d'enquêteurs plus fourni. 
littérature sur les expérimentations électorales quant au pourcentage d'électeurs stratèges lors d'un scrutin majoritaire uninominal à deux tours (Baujard et al., 2016, Igersheim et Baujard, 2019). Sans doute ne peut-il cependant être assimilé à la proportion totale d'électeurs qui ont effectué un vote officiel divergeant de leurs préférences absolues sur la base de stratégies électorales diverses. Plusieurs facteurs, en effet, pourraient avoir impliqué chez certains des participants une reproduction du choix stratégique officiel lors du vote alternatif. On peut ainsi envisager que la faible familiarité avec les règles alternatives mène des participants à ne pas forcément s'autoriser à s'affranchir de leur choix officiel, faute de parvenir à extrapoler les résultats alternatifs. Mais il peut aussi s'agir de la volonté, chez ceux qui ont la perception la moins assise de leur "compétence" politique (et donc un sentiment de liberté moindre à l'égard du "jeu" avec la règle électorale), de surafficher à destination des expérimentateurs la stabilité de leurs choix électoraux d'une règle à l'autre, par crainte d'une disqualification de ce qu'ils percevraient comme une forme d'incohérence de leurs préférences civiques entre les scrutins ${ }^{14}$.

La Table 2 reporte les résultats des votes officiels au niveau national, de la Martinique et des bureaux de Dillon D ainsi que les votes officiels indiqués par les participants à l'expérimentation sur leurs bulletins. La Figure 1 donne un aperçu du classement des candidats pour chacun des niveaux afin de favoriser la comparaison.

TABLE 2 - Résultats des votes officiels exprimés (en \%) et classements des candidats

\begin{tabular}{|c|c|c|c|c|c|c|c|c|c|c|c|c|}
\hline \multicolumn{2}{|c|}{ Votes officiels } & 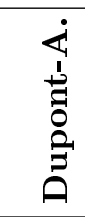 & 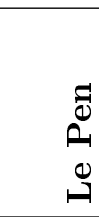 & 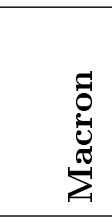 & 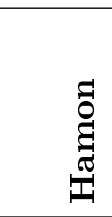 & $\frac{\sigma}{3}$ & $\begin{array}{l}z \\
\stackrel{0}{0} \\
0 \\
0\end{array}$ & 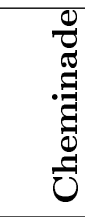 & 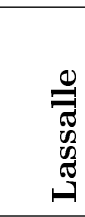 & 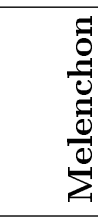 & 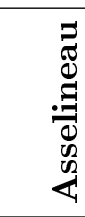 & 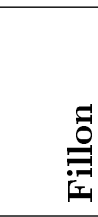 \\
\hline \multirow{2}{*}{ National } & Score & 4,70 & 21,30 & 24,01 & 6,36 & 0,64 & 1,09 & 0,18 & 1,21 & 19,58 & 0,92 & 20,01 \\
\hline & rang & 6 & 2 & 1 & 5 & 10 & 8 & 11 & 7 & 4 & 9 & 3 \\
\hline \multirow{2}{*}{ Martinique } & Score & 2,14 & 10,94 & 25,53 & 9,76 & 2,06 & 2,94 & 0,34 & 0,80 & 27,37 & 1,29 & 16,84 \\
\hline & rang & 7 & 4 & 2 & 5 & 8 & 6 & 11 & 10 & 1 & 9 & 3 \\
\hline \multirow{2}{*}{ Bureaux Dillon D } & Score & 2,16 & 9,80 & 23,62 & 15,81 & 2,32 & 3,90 & 0,26 & 0,74 & 29,20 & 1,16 & 11,02 \\
\hline & rang & 8 & 5 & 2 & 3 & 7 & 6 & 11 & 10 & 1 & 9 & 4 \\
\hline \multirow{2}{*}{ Participants à̀ } & Score & 2,67 & 7,56 & 24,00 & 15,78 & 2,00 & 6,00 & 0,22 & 0,00 & 31,55 & 1,78 & 8,44 \\
\hline & rang & 7 & 5 & 2 & 3 & 8 & 6 & 10 & 11 & 1 & 9 & 4 \\
\hline
\end{tabular}

14. Il convient en effet de ne pas sous-estimer les manifestations, dans le cadre de ce type d'expérimentation, de mécanismes d'inhibition vis-à-vis des expérimentateurs assimilables à ceux qui sont désormais bien documentés pour les sondages d'opinion (Gaxie, 1990). Même si le scrutin est anonyme, en effet, et si le face-à-face avec le chercheur est médiatisé par le bulletin, le caractère peu routinisé du scrutin et son insertion dans une recherche universitaire peuvent mener à des effets d'autocensure qu'il convient de garder à l'esprit. 
FiguRE 1 - Classements des candidats sur la base des résultats officiels

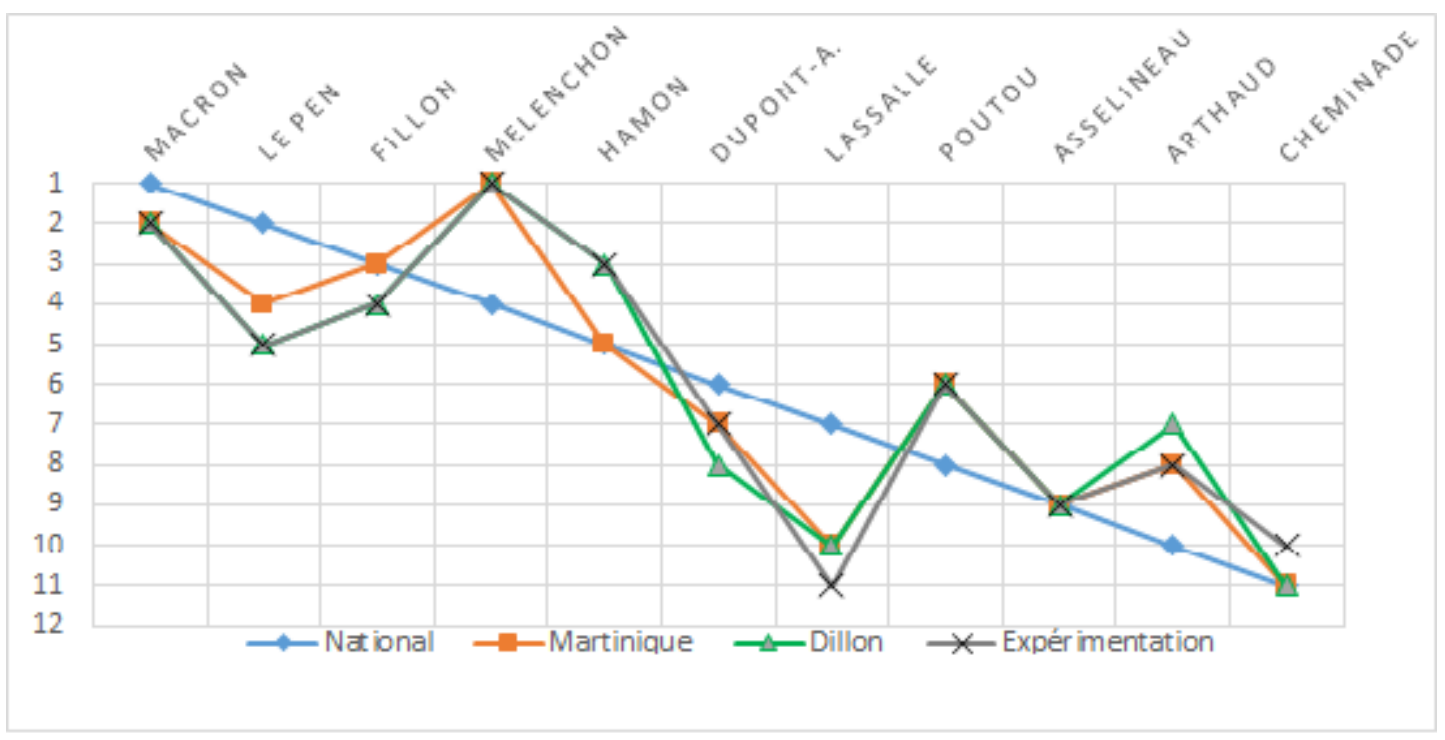

Le classement obtenu sur la base des réponses à la question concernant le vote officiel est le même que celui obtenu sur l'ensemble des bureaux de Dillon en ce qui concerne les six candidats de tête. Quelques inversions s'opèrent ensuite entre les candidats ayant obtenu entre 0 et $3 \%$ des voix. L'échantillon des participants à l'expérimentation est en tout cas très représentatif de la population des électeurs officiels des bureaux concernés du point de vue de la hiérarchie entre candidats opérée à travers le vote. Et les écarts en pourcentages sont relativement faibles, échelonnés entre 0,03 et 2, 58\% en négatif (sous-représentation des électeurs déclarés du candidat par rapport au vote officiel dans les bureaux) et entre 0,38 et $2,1 \%$ en positif (sur-représentation), ce qui démarque notre expérimentation de celles qui ont été réalisées ailleurs en France à l'occasion de ce scrutin. Dans les autres villes, en effet, il a pu être observé chez les participants à l'expérimentation ${ }^{15}$ une sous-représentation systématique et forte des votants officiels pour M. Le Pen et F. Fillon, allant pour la première jusqu'à un écart de 6,93\% à Strasbourg, et pour le second de 8,7\% à Grenoble, tandis que les votants officiels Hamon et Mélenchon étaient surreprésentés à des hauteurs pouvant aller jusqu'à 5 ou $6 \%$. Deux hypothèses, sans doute conjointes, peuvent être avancées pour expliquer ce type de distorsion. La première est que les électeurs ont diversement joué le jeu de l'expérimentation en fonction de leur vote officiel. Les électeurs ancrés à gauche ont peut-être davantage été portés à tester les alternatives parce qu'ils étaient moins satisfaits de la règle majoritaire à deux tours dans le contexte de 2017. La seconde hypothèse - puisque le vote officiel n'est pas identifié grâce à une donnée officielle mais au travers de la mention déclarative qui en est faite par l'expérimentateur sur son bulletin - est qu'on observe un effet, bien connu des sondeurs, de sous-déclaration de votes perçus comme moins assumables socialement. Il s'agirait en l'espèce des votes Fillon - dans le contexte de l'affaire dont on sait qu'elle a terni l'image

15. Voir le compte-rendu sur https://www.gate.cnrs.fr/spip.php?article580. Le lecteur peut également se référer au rapport complet sur les expérimentations menées en France Hexagonale en 2017 via le lien https://zenodo.org/record/1199545\#.W304dH4yVEI. 
du candidat LR et contribué à son mauvais score - et Le Pen - d'une manière qui contredit pour le coup la tendance à l'effacement de la sous-déclaration du vote FN qui a été observée par les sondeurs à la suite de la succession opérée entre Jean-Marie Le Pen et sa fille, mais dans un contexte local (ville de gauche) qui permet pour autant de maintenir cette seconde hypothèse.

Quoi qu'il en soit, les électeurs des bureaux de Dillon ayant participé à l'expérimentation sont, eux, beaucoup plus représentatifs des votes officiels dans les bureaux concernés. Soit qu'ils aient joué le jeu de l'expérimentation indépendamment de leurs préférences officielles pour les candidats, soit qu'ils assument davantage leur vote, dans un contexte où les voix Le Pen et Fillon sont de toute façon plutôt faibles par rapport au niveau national. On ne peut pour autant écarter complètement l'hypothèse de déclarations non conformes au vote officiel réel et cette limite à l'extrapolation des résultats mérite d'être gardée à l'esprit pour l'analyse. On observe du reste que les écarts négatifs les plus importants sont bien enregistrés, là aussi même si dans une moindre mesure, pour les candidats Fillon et Le Pen.

Au niveau de la Martinique, le classement est identique au classement officiel de Dillon seulement jusqu'au deuxième candidat. Au niveau national, les classements divergent avec tous les niveaux dès le candidat en tête et ne se rejoignent que ponctuellement, tant avec la Martinique qu'avec Dillon. C'est ainsi qu'E. Macron, arrivé en tête au niveau national, se trouve devancé par J.-L. Mélenchon, 4è national et ler local, sur les autres niveaux. Sa rivale au second tour, M. Le Pen, n'occupe quant à elle que la quatrième position en Martinique, et la cinquième dans nos bureaux, officiellement comme expérimentalement. Les candidats Poutou et Arthaud occupent un rang de classement local supérieur à celui que leur octroie le niveau national. La tendance au vote plus à gauche que la moyenne nationale est donc maintenue en Martinique, et accentuée à Dillon, même si elle est davantage brouillée que dans les élections présidentielles précédentes par la candidature d'E. Macron et par les déboires du candidat socialiste, malgré tout classé en 3e position à Dillon. La somme des votes pour les candidats Mélenchon, Hamon, Poutou et Arthaud atteint ainsi 27,67\% au niveau national, 42,13\% en Martinique, 51,23\% à Dillon et 55,33\% chez nos électeurs participants à l'expérimentation.

\subsection{Biais de représentativité et de participation}

Du fait que les participants à l'expérimentation ne représentent qu'une partie des votants des Bureaux de Dillon D et qu'ils n'ont pas tous dévoilé leur choix au scrutin officiel, les résultats bruts contiennent des biais de représentativité et de participation. Il s'ensuit que dans notre échantillon expérimental, certains candidats se retrouvent surreprésentés comparativement à d'autres qui sont plutôt sous-représentés. La nécessité de corriger ce biais s'impose dès lors si l'on envisage une comparaison entre les résultats et les classements expérimentaux et ceux du scrutin officiel. En effet, les règles de vote expérimentées au niveau local se déroulent toutes les deux en un seul tour, contrairement au scrutin officiel. Or, le second tour de ce dernier n'a pas opposé les deux candidats arrivés en tête du premier tour au niveau local. Nous disposons donc d'un résultat expérimental localisé et d'un résultat officiel uniquement au niveau national. La comparaison des résultats du vote officiel avec le vote expérimental en termes de désignation du vainqueur n'est donc permise qu'en redressant les résultats de ce dernier pour rendre notre échantillon représentatif du vote national. 
Décrivons comment nous procédons en vue de corriger le biais.

Des 508 bulletins exploitables, nous n'en garderons que 450, qui correspondent à ceux pour lesquels l'électeur mentionne avoir voté pour un candidat; nous excluons donc les 47 bulletins "blanc" et les 11 bulletins "sans réponse". Sur la base des 450 bulletins valablement exprimés, nous obtenons les chiffres de la quatrième ligne de la Table 2. En comparant ces chiffres à ceux des lignes qui précèdent, nous avons un aperçu de la sur-représentativité ou sous-représentativité de certains résultats selon que l'on se projette au niveau des bureaux de Dillon D, de la Martinique ou surtout de la France entière.

$\mathrm{Si}$, à des fins de comparaison des règles de vote en termes de désignation du vainqueur de l'élection, on se situe au niveau national, on remarque que dans notre échantillon expérimental, les électeurs de B. Hamon, P. Poutou, J.-L. Mélenchon et de N. Arthaud sont surreprésentés, quand ceux de N. Dupont-Aignan, M. Le Pen et de F. Fillon sont sousreprésentés. Il y a en revanche un certain équilibre en ce qui concerne les électeurs des trois autres candidats. Pour mettre en œuvre la correction du biais au niveau national, on accordera donc à un électeur ayant voté pour un candidat $X$, un poids égal au rapport entre le pourcentage de votes exprimés obtenu(s) lors du vote officiel national et le pourcentage de votes officiels déclaré(s) par les participants à l'expérimentation. Cette méthode de correction des biais est simple et intuitive, même si non exempte de critiques. Elle a été retenue pour l'ensemble des expérimentations locales menées lors de l'élection présidentielle française de $2017^{16}$. Notons qu'une limite immédiate qu'elle présente pour notre expérimentation est que dans la mesure où J. Lassalle n'a obtenu aucune voix officielle parmi les électeurs ayant participé à l'expérimentation, aucun poids ne peut lui être associé.

La Table 3 reprend les poids affectés aux électeurs en fonction du candidat pour lesquels ils ont voté.

TABLE 3 - Poids associés aux électeurs de chaque candidat

\begin{tabular}{|c|c|c|c|c|c|c|c|c|c|c|c|}
\hline & 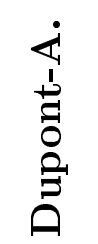 & $\begin{array}{l}\tilde{0} \\
0 \\
\rho_{1} \\
\stackrel{0}{\oplus}\end{array}$ & 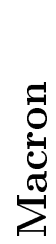 & 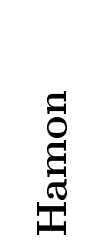 & $\frac{\sigma}{\tilde{\sigma}}$ & $\begin{array}{l}\vec{J} \\
0 \\
\stackrel{+}{Z} \\
0 \\
0_{1}\end{array}$ & 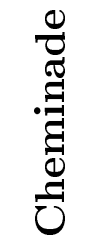 & 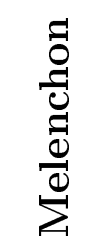 & 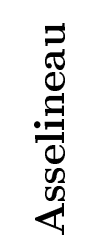 & 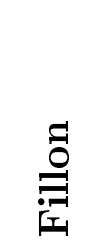 & 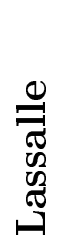 \\
\hline Poids & 1,76 & 2,82 & 1 & 0,40 & 0,32 & 0,18 & 0,82 & 0,62 & 0,52 & 2,37 & - \\
\hline
\end{tabular}

Uniquement à titre de comparaison, nous fournissons dans l'Annexe C, les résultats des deux règles de vote (sans les comparaisons par paires) si l'on avait eu recours à un redressement basé sur les résultats de la Martinique. Cette présentation fait suite à une recommandation d'un des rapporteurs motivée par le fait que corrélation avec les résultats nationaux est de 0,77 alors que celle de la Martinique de 0,93. Nous n'ouvrons pas ici le débat sur la "meilleure" façon de traiter les biais. Il est vrai que le choix de la référence de redressement est en soi une tout autre question sur laquelle la littérature devrait se pencher afin d'ouvrir

16. Nous remercions Isabelle Lebon et Antoinette Baujard pour les discussions sur la manière de corriger le biais. 
une discussion scientifique poussée. Une telle discussion servira alors de guide pour les futures expérimentations.

\subsection{Les résultats avec la règle du Vote Alternatif}

Rappelons que la règle du vote alternatif exige de l'électeur de classer tout ou partie des candidats (au moins un). Des 508 bulletins exploitables pour l'expérimentation, 505 (soit $99,41 \%)$ ont indiqué un classement valide pour le vote alternatif. Lors de cette expérimentation, nous avons demandé aux électeurs de classer tous les candidats. Certains électeurs ont clairement exprimé la difficulté de l'exercice, mais une très large majorité d'entre eux (96, 24\%) s'y est pliée, ce qui suggère, à l'instar de Farvaque et al. (2011), que la nécessité de classer tous les candidats ne constitue pas un obstacle insurmontable.

La Table 4 reporte les résultats des candidats après chaque itération. Une case noircie à une itération donnée indique que le candidat a été éliminé lors de l'itération précédente. Le transfert de votes se lit aisément dans cette table : une fois un candidat éliminé à un tour $T_{j}$, il suffit de faire la différence des voix entre les tours $T_{j+1}$ et $T_{j}$ pour savoir comment sont transférées les voix du candidat éliminé.

TABLE 4 - Itérations du vote alternatif

\begin{tabular}{|c|c|c|c|c|c|c|c|c|c|c|c|}
\hline & \multicolumn{10}{|c|}{ Itérations } & \\
\hline Candidats & T1 & T2 & T3 & $\mathrm{T4}$ & T5 & T6 & T7 & T8 & T9 & T10 & Class. \\
\hline Cheminade & 0 & & & & & & & & & & 11 \\
\hline Lassalle & 4 & 4 & & & & & & & & & 10 \\
\hline Asselineau & 7 & 7 & 7 & & & & & & & & 9 \\
\hline Arthaud & 13 & 13 & 13 & 13 & & & & & & & 8 \\
\hline Dupont-A. & 20 & 20 & 20 & 21 & 22 & & & & & & 7 \\
\hline Poutou & 25 & 25 & 26 & 27 & 29 & 31 & & & & & 6 \\
\hline Le Pen & 38 & 38 & 38 & 39 & 39 & 44 & 48 & & & & 5 \\
\hline Fillon & 45 & 45 & 45 & 45 & 45 & 49 & 49 & 58 & & & 4 \\
\hline Hamon & 78 & 78 & 78 & 80 & 85 & 88 & 94 & 101 & 112 & & 3 \\
\hline Macron & 124 & 124 & 127 & 127 & 129 & 134 & 138 & 156 & 189 & 241 & 2 \\
\hline Mélenchon & 151 & 151 & 151 & 153 & 156 & 158 & 175 & 188 & 200 & 259 & 1 \\
\hline
\end{tabular}

J. Cheminade n'étant classé en tête par aucun électeur, il est donc éliminé à la première itération et aucun transfert de voix n'a lieu. Lors de la deuxième itération, c'est J. Lassalle qui est éliminé et un transfert de ses 4 électeurs se fait au profit de P. Poutou $(+1)$ et E. Macron $(+3)$. On note que le candidat F. Fillon ne bénéficie d'aucun transfert entre les itérations T2 et T5. Les seuls transferts dont il bénéficie par la suite, jusqu'à son élimination à la 8e itération proviennent des électeurs ayant voté, à un rang précédent, pour N. Dupont-Aignan, puis M. Le Pen. Les itérations et transferts se poursuivent jusqu'à l'élimination de M. Le Pen à la septième itération; les voix de $\mathrm{M}$. Le Pen sont transférées de la manière suivante : 9 voix vers F. Fillon, 7 voix vers B. Hamon, 13 voix vers J.-L. Mélenchon et 18 voix vers E. Macron. Au terme des itérations, on note que J.-L. Mélenchon sort vainqueur du duel final 
qui l'oppose à E. Macron. La dernière colonne de la Table 4 nous donne le classement final brut : J.-L. Mélenchon arrive en tête suivi de E. Macron puis de B. Hamon, F. Fillon, M. Le Pen, P. Poutou, etc..

Notons qu'en plus d'être le vainqueur de la règle du vote alternatif, J.-L. Mélenchon est aussi le vainqueur de Condorcet. Ce terme renvoie à un candidat majoritairement préféré à chacun des autres lors d'un face à face électoral (Condorcet, 1785). Le fait de favoriser le vainqueur de Condorcet tend à être érigé dans la littérature en critère d'évaluation du respect des préférences des électeurs et donc de la performance d'une règle de vote (voir Farvaque et al., 2009). En l'occurrence, J.-L. Mélenchon battrait chacun des autres candidats en duels majoritaires. Les résultats de tous les duels majoritaires sont consignés dans la Table 8 en Annexe. De cette table, on note également que M. Le Pen est le perdant de Condorcet dans la mesure où, avec notre échantillon de votants, elle serait battue par chacun des autres candidats en duels majoritaires. E. Macron ne perdrait qu'un seul duel, celui face à J.-L. Mélenchon; B. Hamon en perdrait deux (face à E. Macron et J.-L. Mélenchon). F. Fillon perdrait tous ses duels, excepté face à M. Le Pen. P. Poutou ne perdrait ses duels qu'en face de E. Macron, J.-L. Mélenchon et B. Hamon. L'ensemble de ces duels manifeste bien, ainsi, l'ancrage à gauche des électeurs de nos bureaux de vote, ainsi que leur aversion pour le vote FN.

Comme nous l'avons souligné plus haut, nos résultats bruts ne peuvent être extrapolés du fait du biais de représentativité et de participation. En utilisant les poids donnés dans la Table 3, les calculs corrigés pour l'ensemble des itérations sont consignés dans la Table 5.

TABLE 5 - Itérations du vote alternatif avec correction

\begin{tabular}{|c|c|c|c|c|c|c|c|c|c|c|c|}
\hline & \multicolumn{10}{|c|}{ Itérations } & \\
\hline Candidats & T1 & T2 & T3 & T4 & T5 & T6 & T7 & T8 & T9 & T10 & Class. \\
\hline Cheminade & 0 & & & & & & & & & & 11 \\
\hline Lassalle & 1,4 & 1,4 & & & & & & & & & 10 \\
\hline Asselineau & 3,12 & 3,12 & 3,12 & & & & & & & & 9 \\
\hline Arthaud & 3,36 & 3,36 & 3,36 & 3,36 & & & & & & & 8 \\
\hline Poutou & 4,53 & 4,53 & 4,53 & 5,05 & 5,37 & & & & & & 7 \\
\hline Dupont-A, & 29,63 & 29,63 & 29,63 & 30,15 & 30,47 & 30,83 & & & & & 6 \\
\hline Hamon & 30,57 & 30,57 & 30,57 & 31,61 & 33,37 & 34,31 & 38,86 & & & & 5 \\
\hline Fillon & 86,21 & 86,21 & 86,21 & 86,21 & 86,21 & 86,21 & 92,62 & 93,42 & & & 4 \\
\hline Le Pen & 87,73 & 87,73 & 87,73 & 88,25 & 88,25 & 88,79 & 93,49 & 94,69 & 115,41 & & 3 \\
\hline Melenchon & 104,42 & 104,42 & 104,42 & 104,94 & 105,58 & 108,93 & 110,69 & 127,2 & 147,06 & 191,44 & 2 \\
\hline Macron & 106,66 & 106,66 & 108,06 & 108,06 & 108,38 & 108,56 & 115,63 & 131,69 & 183,92 & 252,93 & 1 \\
\hline
\end{tabular}

Notons tout de même que J. Lassalle, bien que n'ayant obtenu aucune voix dans le scrutin officiel parmi les électeurs ayant participé à l'expérimentation, est néanmoins classé en tête par quatre électeurs ayant voté pour un autre candidat au scrutin officiel. Du fait des poids de ces quatre électeurs, un redressement s'opère pour ce candidat. Les Tables 4 et 5 s'accordent sur l'ordre des quatre premiers candidats éliminés. Avec la correction, c'est P. Poutou qui 
est éliminé lors de la cinquième itération; N. Dupont-Aignan est maintenant éliminé lors de la sixième itération, M. Le Pen lors de la neuvième. Le duel final oppose toujours E. Macron à J.-L. Mélenchon. Mais cette fois-ci, c'est E. Macron qui est le vainqueur. La Figure 2 permet de comparer les classements bruts et corrigés des candidats avec le classement national officiel.

FiguRE 2 - Vote Alternatif et Classements des candidats

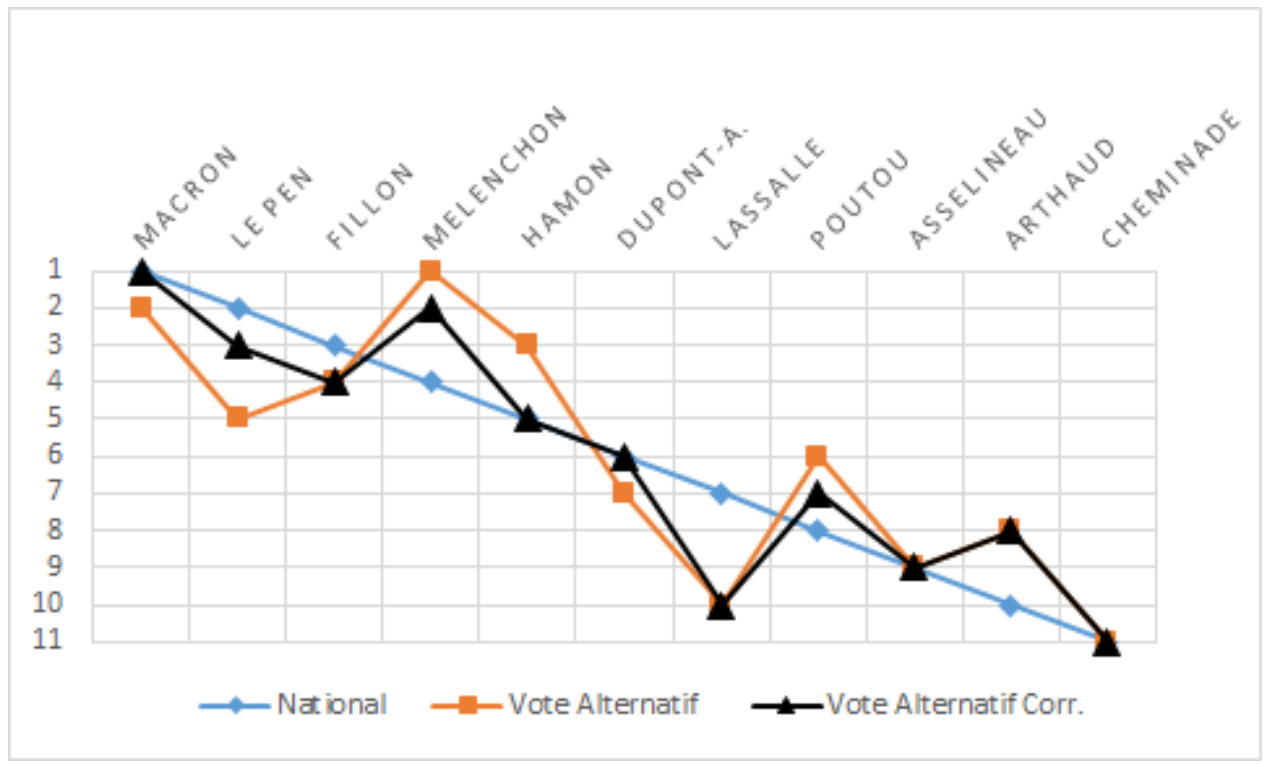

Le résultat final corrigé de l'expérimentation est donc conforme au résultat du vote officiel : le même vainqueur est désigné. En revanche, les rangs de classement des candidats suivants diffèrent assez largement, essentiellement en raison de la présence de J.-L. Mélenchon à la deuxième place expérimentale, au lieu de la quatrième officielle, les autres "gros" candidats se succédant derrière lui dans l'ordre où ils se suivent dans le vote officiel. Compte tenu de la faible taille de l'échantillon et de l'utilisation impérieuse d'une méthode de redressement, il est difficile de monter en généralité à partir de ce résultat, qui ne peut pas être considéré comme un reflet fidèle de ce qu'aurait donné la proposition de la règle de vote alternatif aux citoyens français en 2017. La concordance exacte entre les classements issus du vote officiel indiqué par les participants à l'expérimentation et de leur vote expérimental vérifie du reste la conclusion de l'expérimentation de 2007 quant à la forte proximité entre la règle du vote alternatif et le scrutin majoritaire (Farvaque et al., 2009). En revanche, à la différence de ce qui est observé dans ce travail, cette règle de vote semble bien favoriser le vainqueur de Condorcet en 2017.

La Table 10 en Annexe reporte les résultats des comparaisons par paires lorsqu'on prend en compte la correction. Les résultats avec et sans correction sont assez différents. Avec la correction, E. Macron devient le vainqueur de Condorcet tandis que F. Asselineau devient le perdant de Condorcet. On note également que M. Le Pen ne remporte qu'un seul duel, celui contre F. Asselineau; J.-L. Mélenchon ne perdrait qu'un seul duel, celui face à E. Macron; B. 
Hamon serait toujours battu uniquement par E. Macron et J.-L. Mélenchon. Cette fois-ci, F. Fillon ne perdrait que ses duels face à B. Hamon, E. Macron et J.-L. Mélenchon. P. Poutou ne perdrait ses duels qu'en face de E. Macron, J.-L. Mélenchon et B. Hamon.

\subsection{Les résultats avec la règle de Borda à 4}

Pour rappel, la règle de Borda à 4 exige que chaque électeur classe dans un ordre strict quatre candidats (du plus préféré au moins préféré). Chaque fois qu'un candidat sera classé premier, il recevra 4 points; il recevra 3 points toutes les fois qu'il sera classé deuxième; 2 points lorsqu'il sera classé troisième; 1 point lorsqu'il sera classé quatrième. Il ne recevra aucun point lorsqu'il n'est pas classé. Le score d'un candidat sera égal à la somme totale des points reçus.

Notons que des 508 bulletins exploitables pour l'expérimentation, 491 votants (soit 96, 65\%) ont établi un classement valide pour la règle de Borda à 4. Dans la Table 6, nous reportons la répartition des rangs occupés par les différents candidats selon les classements des électeurs. Ensuite, nous calculons les scores bruts et en déduisons le classement collectif. Sur la base des scores bruts, J.-L. Mélenchon arrive en tête avec un score de 1111 points; il est suivi par E. Macron avec 979 points. B. Hamon (852 points) arrive en troisième position, suivi de F. Fillon (431 points) puis de M. Le Pen (383 points). J. Cheminade occupe la queue du classement avec 84 points.

TABLE 6 - Classements et scores de Borda à 4

\begin{tabular}{|c|c|c|c|c|c|c|c|c|c|c|c|c|}
\hline \multicolumn{13}{|c|}{ Candidats } \\
\hline & & 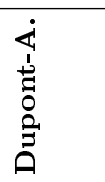 & 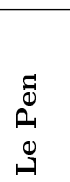 & 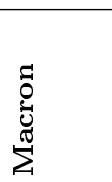 & $\begin{array}{l}\tilde{\Xi} \\
\text { छ్ } \\
\text { త్ర }\end{array}$ & 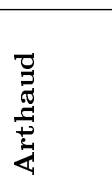 & $\begin{array}{l}\tilde{\partial} \\
\stackrel{0}{0} \\
0 \\
0\end{array}$ & 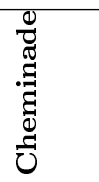 & 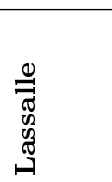 & 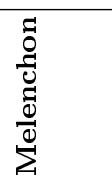 & 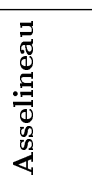 & $\stackrel{\Xi}{\stackrel{\Xi}{\rightleftarrows}}$ \\
\hline \multirow{4}{*}{ Rangs } & 1 & 16 & 40 & 125 & 76 & 13 & 21 & 0 & 3 & 144 & 6 & 47 \\
\hline & 2 & 15 & 41 & 94 & 98 & 21 & 58 & 11 & 9 & 95 & 9 & 40 \\
\hline & 3 & 32 & 28 & 72 & 93 & 43 & 36 & 12 & 20 & 101 & 22 & 32 \\
\hline & 4 & 35 & 44 & 53 & 68 & 54 & 44 & 27 & 40 & 48 & 19 & 59 \\
\hline \multicolumn{2}{|c|}{ Scores bruts } & 208 & 383 & 979 & 852 & 255 & 374 & 84 & 119 & 1111 & 114 & 431 \\
\hline \multicolumn{2}{|c|}{ Classement brut } & 8 & 5 & 2 & 3 & 7 & 6 & 11 & 9 & 1 & 10 & 4 \\
\hline \multirow{2}{*}{\multicolumn{2}{|c|}{ Scores corrigés }} & 222,82 & 560 & 844,88 & 592,65 & 158,09 & 214,25 & 71,81 & 119,07 & 822,94 & 86,71 & 579,18 \\
\hline & & 6 & 5 & 1 & 3 & 8 & 7 & 11 & 9 & 2 & 10 & 4 \\
\hline
\end{tabular}

Dans la Table 6, nous avons également calculé les scores corrigés pour tenir compte du biais de participation et de représentativité. Sur cette base, c'est E. Macron qui est le vainqueur de la règle de Borda à 4 avec 844,88 points ; il est suivi de près par J.-L. Mélenchon (822, 94 points). Comparativement au classement brut, B. Hamon, F. Fillon, M. Le Pen, J. Cheminade, F. Asselineau et J. Lassalle conservent leur place tandis que N. Dupont-Aignan gagne deux places (passant de huitième à sixième) ; N. Arthaud et P. Poutou perdent chacun une place. La Figure 3 donne une représentation des résultats permettant de comparer le classement officiel à ceux de la règle de Borda à 4 . 
FiguRE 3 - Borda à 4 et Classements des candidats

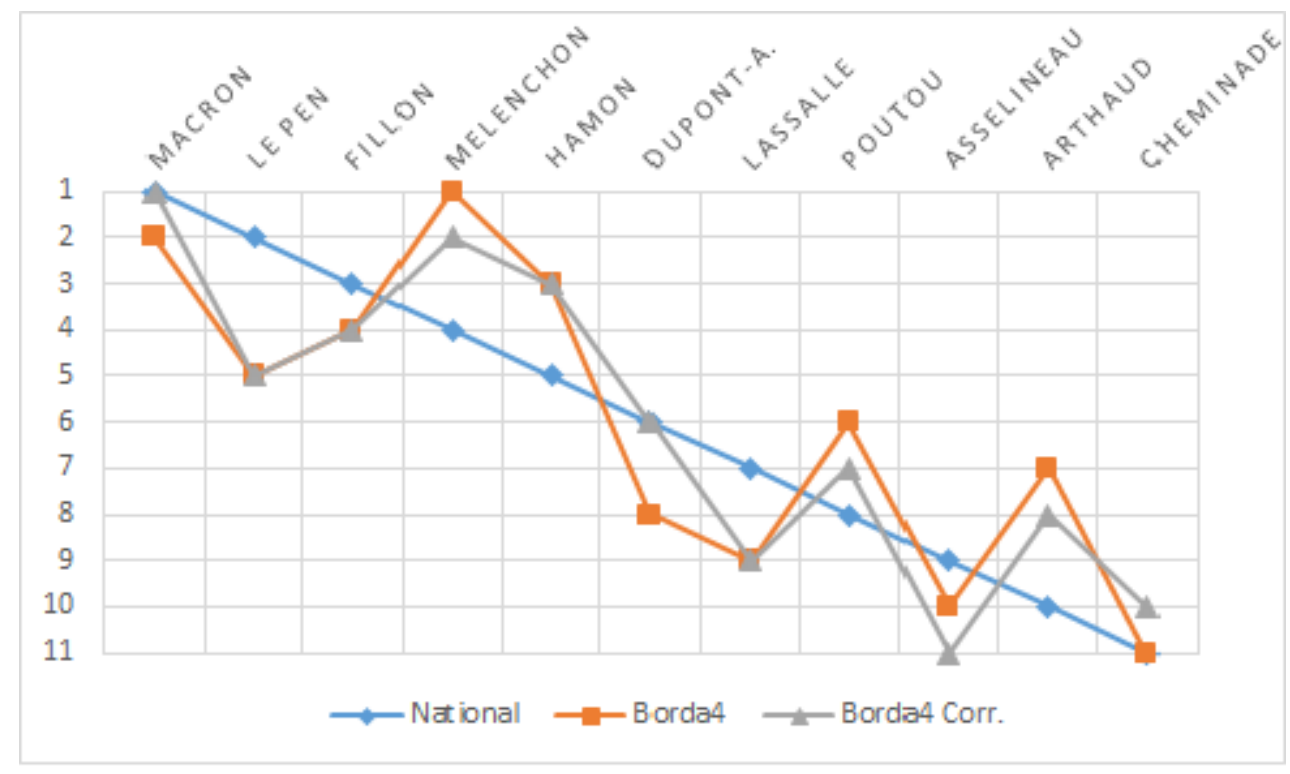

Sur la base des données brutes, la Table 9 en Annexe rend compte des résultats des duels majoritaires. De cette table, on note que J.-L. Mélenchon est le vainqueur de Condorcet et il est l'unique candidat qui défait en duel E. Macron. M. Le Pen apparaît être le perdant de Condorcet. La Table 11 quant à elle rend compte des comparaisons corrigées des biais. On y note des différences avec les résultats bruts : E. Macron devient le vainqueur de Condorcet et le seul qui défait J.-L Mélenchon; J. Cheminade est le perdant de Condorcet. M. Le Pen est battu par E. Macron, J.-L Mélenchon, B. Hamon et F. Fillon.

\subsection{Les électeurs et les règles de vote}

Puisqu'il s'agissait, dans notre cas, pour les deux règles de vote expérimentales de classer les candidats par ordre de préférence, il paraissait intéressant de vérifier dans un premier temps si les classements pour les quatre premiers candidats étaient les mêmes quelle que soit la règle. Pour plus de 90,8\% des électeurs ayant classé au moins 4 candidats, le classement est effectivement le même sous Borda à 4 et le vote alternatif. Ce résultat traduit la cohérence et la sincérité des choix d'une large majorité des électeurs lors de l'expérimentation, du moins pour ce qui concerne les premiers candidats classés. Par conséquent, l'instruction de classer tous les candidats, même si elle n'apparaît pas insurmontable, n'apparaît pas non plus d'une impérieuse nécessité au regard des résultats obtenus. Pour les $9,2 \%$ ayant classé différemment leurs quatre premiers candidats, il est plausible qu'au-delà d'un ou deux candidats connus de certains électeurs ou déjà identifiés comme étant les seuls à pouvoir recueillir leur vote, le classement se fasse de manière aléatoire.

Lors de l'expérimentation la première question posée à l'électeur sur son bulletin était celle de savoir s'il était satisfait de la règle de vote actuelle pour l'élection du président de la République. Le dépouillement des réponses révèle que sur les 508 bulletins exploitables, 
304 (soit 59, 84\%) électeurs se disent satisfaits de la règle actuelle, 178 (soit 35,04\%) ne le sont pas et 26 (soit $5,15 \%$ ) ne se sont pas prononcés. Toutefois, si l'on distingue la réponse à cette question en fonction de la catégorie socio-professionnelle (CSP), il s'avère que "seuls" $48 \%$ des électeurs appartenant à la CSP "Cadres/professions intellectuelles supérieures" sont pleinement satisfaits de la règle de vote actuelle. Pour toutes les autres CSP, les taux de satisfaction sont eux compris entre $60 \%$ et $70 \%$. Ce résultat peut être mis en rapport avec les travaux sur la "compétence" politique légitime (Gaxie, 1978). On peut en effet supposer que les électeurs appartenant aux catégories socio-professionnelles les plus favorisées - corrélées, via leur plus haut niveau d'études principalement, à la détention de cette "compétence"- ont une connaissance objective des modes de scrutin leur permettant plus facilement d'identifier les limites de la règle majoritaire à deux tours, mais aussi que le sentiment subjectif de compétence qu'ils entretiennent les porte à s'estimer, davantage que les électeurs des catégories moins favorisées, fondés à dénoncer le mode de scrutin existant dans le cadre d'une telle expérimentation. La faiblesse de notre échantillon ne permet pas de faire une analyse de régression de ce facteur mais il apparaîtrait intéressant de l'interroger dans une future enquête de plus vaste ampleur.

Après avoir testé une (ou les) règles expérimentale(s), la dernière question posée aux participants de l'expérimentation était de signifier laquelle des règles (officielle, vote alternatif ou Borda à 4) leur paraissait la plus satisfaisante. Pour mieux rendre compte des avis des électeurs, nous nous sommes focalisés sur les 458 électeurs ayant testé les deux règles expérimentales. La répartition des avis est donnée dans la Table 7.

TABLE 7 - Répartition des avis sur les règles de vote

\begin{tabular}{|c|l|c|c|c|c|c|}
\cline { 3 - 6 } \multicolumn{2}{c|}{} & \multicolumn{4}{c|}{ Règle la plus satisfaisante } & \multirow{2}{*}{ Total } \\
\cline { 3 - 7 } \multicolumn{2}{c|}{} & Actuelle & Vote Alternatif & Borda à 4 & Sans réponse & \\
\hline \multirow{2}{*}{$\begin{array}{c}\text { Satisfaction } \\
\text { règle actuelle }\end{array}$} & Sans réponse & 9 & 3 & 7 & 5 & 24 \\
\cline { 2 - 7 } & Oui & 144 & 38 & 72 & 17 & 271 \\
\cline { 2 - 7 } & Non & 22 & 41 & 93 & 7 & 163 \\
\hline \multicolumn{2}{|l|}{ Total } & 175 & 82 & 172 & 29 & 458 \\
\hline
\end{tabular}

Sur la base des 271 électeurs qui se disaient satisfaits de la règle actuelle, 144 (soit $53,14 \%$ ) le demeurent tandis que 72 (soit 26,57\%) préfèrent la règle de Borda à 4 et 38 (soit $14,02 \%)$ le vote alternatif. Parmi les 163 électeurs se disant non satisfaits de la règle actuelle, 93 (soit 57,06\%) préfèrent la règle de Borda à 4, 41 (soit 25, 15\%) préfèrent le vote alternatif et 22 (soit 13,49\%) optent finalement pour la règle actuelle. Il apparaît donc que sur les 458 électeurs ayant testé les deux règles, seuls 175 (soit 38,21\%) trouvent la règle actuelle plus satisfaisante contre 254 (soit 55,46\%) qui préfèrent l'une des règles expérimentales. Si l'on compare les règles séparément, la règle actuelle $(38,21 \%)$ arrive en tête suivie de très près par la règle de Borda à $4(37,55 \%)$. Le vote alternatif ne récolte que $17,91 \%$ de soutien. Ce dernier résultat s'explique aisément par la difficulté qu'ont rencontré de nombreux électeurs à classer le nombre relativement élevé de candidats. 


\section{Conclusion}

Comme le notent Laslier (2019, 2012), Igersheim et Baujard (2019), les théoriciens du vote sont quasi tous unanimes à reconnaître les lacunes des scrutins uninominaux et en particulier la piètre qualité de la règle majoritaire. Un consensus est loin d'être trouvé sur la "meilleure" règle vers laquelle se tourner. L'objet de l'expérimentation étant de confronter le comportement effectif des votants aux prédictions théoriques, l'un des buts poursuivis est l'éducation ou la vulgarisation des possibles règles alternatives au système actuel. L'ambition étant de mieux faire comprendre au citoyen la portée d'une règle de vote, son implémentation, ses avantages et faiblesses (ceci en comparaison avec le vote uninominal pour ce qui est du cas de la France).

Dans cet article, nous avons présenté les résultats de la première expérimentation de deux modes de scrutin, la règle de Borda à 4 et le vote alternatif, dans un territoire d'outremer français, la Martinique, à l'occasion de l'élection présidentielle de 2017. Le but de cette expérimentation était double. Premièrement, il s'agissait d'apporter quelques éléments de réponse à la question de savoir si un autre mode de scrutin serait préférable à la règle actuelle pour les électeurs martiniquais particulièrement portés à l'abstention. En Martinique où les partis nationaux sont faiblement représentés parmi les forces politiques locales, le taux d'abstention pour les élections présidentielles est en effet généralement très élevé (60,12\%) à l'élection présidentielle de 2017). Deuxièmement, l'expérimentation permet d'apprécier la mesure dans laquelle le résultat du vote officiel des participants pourrait être affecté si l'une des deux règles alternatives était adoptée. Si les modes alternatifs de vote apparaissent plus satisfaisants que la règle actuelle pour la majorité des électeurs qui les ont testés, la règle de Borda, du fait certainement de sa plus grande simplicité, semble être préférée au vote alternatif. Les résultats bruts de la règle de Borda et du vote alternatif donnent J.-L. Mélenchon vainqueur de cette élection expérimentale, conformément aux votes officiels, alors que la correction des biais de participation et de représentativité place E. Macron en tête pour les deux modes alternatifs de scrutin. Les deux règles de vote testées donnent donc dans notre expérience exactement le même résultat final que celui issu des votes officiels des participants à l'expérimentation.

Toutefois, comme pour toute expérimentation, les résultats obtenus ici doivent être interprétés et généralisés avec prudence du fait, notamment, de la simplicité de la méthode de correction des biais de représentativité et de participation, et des spécificités locales qui pourraient fortement influencer les choix des électeurs. Par ailleurs, même si les règles alternatives de vote ont été longuement expliquées aux participants de l'expérimentation, l'absence d'antériorité de leur application et donc de l'observation de la manière dont elles se traduisent en résultats au niveau collectif, ne leur ont pas permis l'élaboration d'une réelle stratégie de vote, dont la prise en compte pourrait conduire à des résultats complètement différents. Enfin, l'offre politique proposée aux électeurs, elle-même, peut être considérée comme pour partie influencée par la règle de vote en vigueur.

Par-delà, cette première expérimentation ultramarine est porteuse d'un certain nombre d'enseignements méthodologiques utiles. Les aléas de la mise en place du protocole expérimental, tout d'abord, qui n'ont pas permis la diffusion auprès des électeurs d'une information individualisée sur notre expérimentation, ses buts et ses modalités, ainsi que sur les règles de vote alternatives testées, plaident pour un recours impératif à ce dispositif. La participation 
plus faible que dans les autres lieux d'expérimentation que nous avons enregistrée pourrait bien en effet s'expliquer à ce regard. Pour autant, on notera que placer l'électeur en situation de manier stratégiquement les règles de votes supposerait un dispositif d'explication plus poussé encore qu'un simple courrier et passant par des échanges de vive voix très en amont avec les expérimentateurs. Un retour régulier dans les mêmes bureaux de vote pour tester d'une élection à l'autre la même règle et en fournir les résultats aux électeurs irait dans le même sens d'une familiarisation réelle avec ce scrutin alternatif, qui permettrait de recueillir des résultats plus proches d'une situation non expérimentale.

Une autre des limites mise en évidence par notre expérimentation, mais qui sans doute s'y observe sous un jour plus cru et non de manière exclusive aux autres terrains d'enquête, est liée à la large méconnaissance des caractéristiques des électeurs officiels, qui rend difficilement contrôlable la représentativité des participants à l'expérimentation au regard des électeurs du ou des bureaux concernés. La sociologie des comportements électoraux des Martiniquais reste à faire, et les spécificités fortes du vote sur ce territoire attirent l'attention sur cette caractéristique. Mais en réalité, les protocoles expérimentaux, où qu'ils soient mis en place, rendent tout aussi hypothétique cette mesure de représentativité, puisqu'on n'y connaît jamais le profil sociologique de l'ensemble des électeurs ayant voté au scrutin officiel, uniquement celui des participants à l'expérimentation si des questions leur sont posées à ce sujet. Il n'est donc pas possible de savoir si ce second groupe n'est pas caractérisé par certaines distorsions au regard du premier. L'on sait la force des corrélations entre appartenances sociales et participation électorale et les inégalités devant le vote dont elles sont porteuses, qui constituent un acquis désormais solide de la sociologie électorale (pour l'élection concernée, voir Braconnier et al. (2015)). Dès lors, on mesure la difficulté que constitue le fait que ces données sont un point aveugle des expérimentations électorales, si l'on entend tirer des enseignements des expérimentations au sujet de possibles améliorations des modes de scrutin - qui sont un enjeu démocratique au cœur des débats contemporains, comme nous l'avons vu. Le problème est que le recueil de ce type d'information n'est pas possible à l'échelle d'un ou quelques bureaux. Les très riches enquêtes électorales de l'INSEE, en effet, seules données collectées au sujet des caractéristiques des électeurs sur une base réelle et non déclarative ${ }^{17}$, ne sont effectuées qu'à l'échelle régionale et ne peuvent donc être recoupées qu'à ce seul niveau. Enfin, les difficultés d'interprétations de divers résultats ici présentés plaident, comme nous l'avons évoqué, en faveur de la mise en place, dans les protocoles, d'un travail de recueil de données qualitatives permettant notamment d'interroger les motivations des électeurs à voter alternativement, qui pourrait permettre d'enrichir l'analyse. Ces divers enseignements nous semblent montrer combien l'analyse expérimentale in situ a les moyens d'innover encore dans ses dispositifs pour mettre en évidence des ressorts variés des choix électoraux.

17. Elles s'appuient sur la constitution d'un échantillon représentatif pour lequel les listes d'émargement sont consultées après les scrutins, permettant le recueil de données sur la participation et sa régularité d'un tour à l'autre et d'une élection à l'autre, recoupées ensuite avec les données du fichier électoral (âge, genre, commune de naissance) et celles de l'échantillon démographique permanent (état civil, données fiscales, situation salariale). Mises en place en France hexagonale depuis 1984, ces enquêtes sont disponibles pour les départements et régions d'outre-mer depuis 2012, pour les seules élections présidentielles et législatives. 


\section{Annexes}

\section{A. Résultats bruts des comparaisons par paires}

TABLE 8 - Comparaisons par paires sur les classements du Vote Alternatif

\begin{tabular}{|c|c|c|c|c|c|c|c|c|c|c|c|}
\hline & 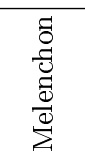 & 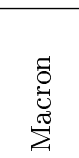 & 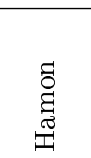 & 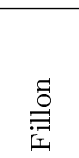 & 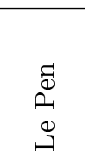 & $\begin{array}{l}\tilde{\sigma} \\
\stackrel{+}{*} \\
\overrightarrow{0} \\
0 .\end{array}$ & $\begin{array}{l}\stackrel{4}{1} \\
\stackrel{1}{\Xi} \\
0 \\
\stackrel{0}{\Xi} \\
\Xi\end{array}$ & 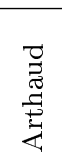 & 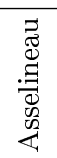 & 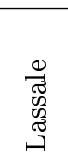 & 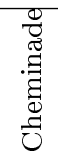 \\
\hline Melenchon & - & 23 & 95 & 224 & 243 & 295 & 324 & 324 & 358 & 340 & 360 \\
\hline Macron & -23 & - & 60 & 205 & 211 & 209 & 249 & 236 & 303 & 291 & 317 \\
\hline Hamon & -95 & -60 & - & 157 & 178 & 202 & 258 & 237 & 287 & 284 & 302 \\
\hline Fillon & -224 & -205 & -157 & - & 27 & 9 & 89 & 53 & 124 & 116 & 134 \\
\hline Le Pen & -243 & -281 & -178 & -27 & - & -11 & 56 & 30 & 97 & 96 & 111 \\
\hline Poutou & -295 & -209 & -202 & -9 & 11 & - & 67 & 47 & 107 & 105 & 116 \\
\hline Dupont-A. & -324 & -249 & -258 & -89 & -56 & -67 & - & -37 & 43 & 28 & 49 \\
\hline Arthaud & -324 & -236 & -237 & -53 & -30 & -47 & 37 & - & 75 & 66 & 81 \\
\hline Asselineau & -358 & -303 & -287 & -124 & -97 & -107 & -43 & -75 & - & -14 & 8 \\
\hline Lassale & -340 & -291 & -284 & -116 & -96 & -105 & -28 & -66 & 14 & - & 18 \\
\hline Cheminade & -360 & -317 & -302 & -134 & -111 & -116 & -49 & -81 & -8 & -18 & - \\
\hline
\end{tabular}

TABLE 9 - Comparaisons par paires sur les classements de la règle de Borda à 4

\begin{tabular}{|c|c|c|c|c|c|c|c|c|c|c|c|}
\hline & 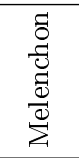 & 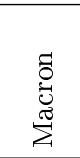 & 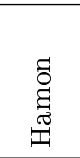 & $\stackrel{\Xi}{\stackrel{\Xi}{\Xi}}$ & 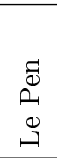 & $\begin{array}{l}\tilde{0} \\
\stackrel{+}{*} \\
0 \\
0\end{array}$ & 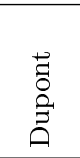 & 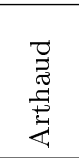 & 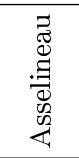 & 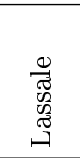 & 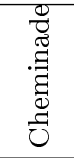 \\
\hline Melenchon & - & 18 & 72 & 266 & 290 & 284 & 338 & 321 & 372 & 327 & 370 \\
\hline Macron & -18 & - & 56 & 297 & 281 & 196 & 251 & 239 & 312 & 276 & 308 \\
\hline Hamon & -72 & -56 & - & 253 & 252 & 222 & 279 & 287 & 339 & 335 & 362 \\
\hline Fillon & -266 & -297 & -253 & - & 129 & -81 & -66 & -51 & -49 & -33 & -31 \\
\hline Le Pen & -290 & -281 & -252 & -129 & - & -188 & -152 & -159 & -112 & -134 & -125 \\
\hline Poutou & -284 & -196 & -222 & 81 & 188 & - & 77 & 40 & 186 & 197 & 209 \\
\hline Dupont & -338 & -251 & -279 & 66 & 152 & -77 & - & -28 & 104 & 49 & 74 \\
\hline Arthaud & -321 & -239 & -287 & 51 & 159 & -40 & 28 & - & 158 & 125 & 170 \\
\hline Asselineau & -372 & -312 & -339 & 49 & 112 & -186 & -104 & -158 & - & -140 & -93 \\
\hline Lassale & -327 & -276 & -335 & 33 & 134 & -197 & -49 & -125 & 140 & - & -35 \\
\hline Cheminade & -370 & -308 & -362 & 31 & 125 & -209 & -74 & -170 & 93 & 35 & - \\
\hline
\end{tabular}

Lecture de la Table : Pour deux candidats $X$ (en ligne) et $Y$ (en colonne), le chiffre à la croisée de la ligne et de la colonne correspond à la différence entre le nombre d'électeurs qui classent le candidat $X$ avant le candidat $Y$ et le nombre d'électeurs qui classent le candidat $Y$ avant le candidat $X$. Si ce nombre est positif, cela indique que le candidat $X$ bat majoritairement le candidat $Y$; s'il est négatif, cela indique que le candidat $Y$ bat majoritairement le candidat $X$. 


\section{B. Résultats corrigés des comparaisons par paires}

TABLE 10 - Comparaisons par paires sur les classements du Vote Alternatif

\begin{tabular}{|c|c|c|c|c|c|c|c|c|c|c|c|}
\hline & 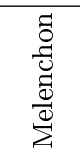 & $\begin{array}{l}\tilde{0} \\
\stackrel{0}{0} \\
\overbrace{\Sigma}^{\pi}\end{array}$ & 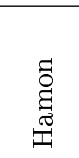 & $\stackrel{\Xi}{\stackrel{\vec{I}}{\mid \vec{I}}}$ & 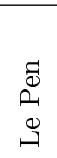 & $\begin{array}{l}\tilde{\Xi} \\
\stackrel{0}{ \pm} \\
0 \\
0\end{array}$ & 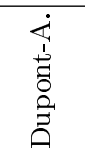 & 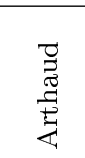 & 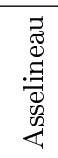 & 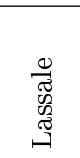 & 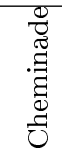 \\
\hline Melenchon & - & -76 & 30 & 110 & 128 & 231 & 232 & 232 & 278 & 203 & 258 \\
\hline Macron & 76 & - & 145 & 166 & 136 & 226 & 216 & 256 & 295 & 260 & 292 \\
\hline Hamon & -30 & -145 & - & 81 & 84 & 213 & 181 & 256 & 276 & 244 & 294 \\
\hline Fillon & -110 & -166 & -81 & - & 77 & 44 & 34 & 54 & 73 & 71 & 75 \\
\hline Le Pen & -128 & -136 & -84 & -77 & - & -22 & -53 & -21 & 7 & -13 & -1 \\
\hline Poutou & -231 & -226 & -213 & -44 & 23 & - & 0,34 & 3 & 137 & 122 & 140 \\
\hline Dupont-A. & -232 & -216 & -181 & -34 & 53 & $-0,34$ & - & 6 & 114 & 58 & 83 \\
\hline Arthaud & -232 & -257 & -256 & -54 & 21 & -3 & -6 & - & 143 & 85 & 131 \\
\hline Asselineau & -278 & -295 & -276 & -73 & -7 & -137 & -114 & -143 & - & -138 & -99 \\
\hline Lassale & -203 & -260 & -244 & -71 & 13 & -122 & -58 & -85 & 138 & - & -31 \\
\hline Cheminade & -258 & -292 & -294 & -75 & 1 & -140 & -83 & -131 & 99 & 31 & - \\
\hline
\end{tabular}

TABLE 11 - Comparaisons par paires sur les classements de la règle de Borda à 4

\begin{tabular}{|c|c|c|c|c|c|c|c|c|c|c|c|}
\hline & 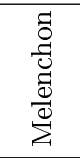 & 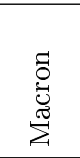 & 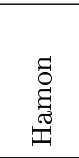 & 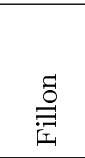 & 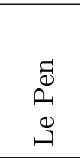 & $\begin{array}{l}\tilde{0} \\
\stackrel{0}{0} \\
0 \\
0\end{array}$ & $\begin{array}{l}\overrightarrow{\tilde{Z}} \\
\stackrel{0}{\Xi} \\
\vec{\Xi}\end{array}$ & 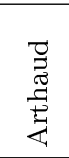 & 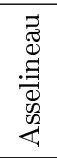 & 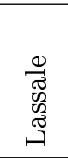 & 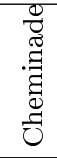 \\
\hline Melenchon & - & -34 & 83 & 80 & 77 & 254 & 216 & 255 & 270 & 244 & 268 \\
\hline Macron & 34 & - & 124 & 97 & 90 & 225 & 207 & 245 & 276 & 255 & 282 \\
\hline Hamon & -83 & -124 & - & 9 & 36 & 180 & 171 & 198 & 225 & 201 & 231 \\
\hline Fillon & -80 & -97 & -9 & - & 12 & 106 & 132 & 126 & 170 & 152 & 172 \\
\hline Le Pen & -77 & -90 & -36 & -12 & - & 98 & 94 & 116 & 145 & 139 & 161 \\
\hline Poutou & -254 & -225 & -180 & -106 & -98 & - & 8 & 25 & 59 & 43 & 63 \\
\hline Dupont & -216 & -207 & -171 & -132 & -94 & -8 & - & 7 & 57 & 27 & 54 \\
\hline Arthaud & -255 & -245 & -198 & -126 & -116 & -25 & -7 & - & 41 & 22 & 42 \\
\hline Asselineau & -270 & -276 & -225 & -170 & -145 & -59 & -57 & -41 & - & -27 & 1 \\
\hline Lassale & -244 & -255 & -201 & -152 & -139 & -43 & -27 & -22 & 27 & - & 24 \\
\hline Cheminade & -268 & -282 & -231 & -172 & -161 & -63 & -54 & -42 & -1 & -24 & - \\
\hline
\end{tabular}

Les chiffres dans les Tables 10 et 11 ont été pour la plupart arrondis à l'entier par souci de simplicité. 


\section{Résultats des règles avec une correction au niveau des résultats de la Martinique}

\section{C.1. Les poids associés aux électeurs}

Lorsqu'on prend comme référence de correction les résultats de la Martinique, on obtient les poids fournis dans la Table 12 .

TABLE 12 - Poids associés aux électeurs de chaque candidat

\begin{tabular}{|c|c|c|c|c|c|c|c|c|c|c|c|}
\hline & 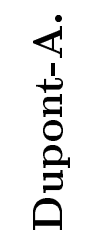 & 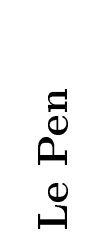 & 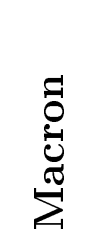 & 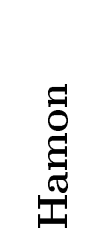 & 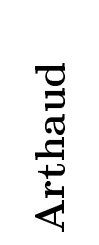 & $\begin{array}{l}\vec{J} \\
0 \\
\stackrel{+}{*} \\
0 \\
0 \\
0_{1}\end{array}$ & 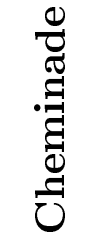 & $\frac{\Xi}{0}$ & 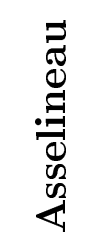 & 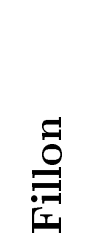 & 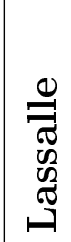 \\
\hline Poids & 0,80 & 1,45 & 1,06 & 0,62 & 1,03 & 0,49 & 1,55 & 0,87 & 0,72 & 1,99 & - \\
\hline
\end{tabular}

Une comparaison des chiffres de la Table 12 et ceux de la Table 3 laisse paraître que M. Le Pen et F. Fillon restent des candidats sous-représentés dans notre échantillon expérimental mais dans une proportion moindre; N. Dupont-Aignan devient un candidat surreprésenté tandis que N. Arthaud et J. Cheminade deviennent sous-représentés. Quant à E. Macron, il reste quelque peu juste-représenté.

\section{C.2. Les résultats du vote alternatif}

La Table 13 fourni les résultats corrigés du vote alternatif.

TABLE 13 - Vote alternatif avec correction niveau Martinique

\begin{tabular}{|c|c|c|c|c|c|c|c|c|c|c|c|}
\hline & \multicolumn{10}{|c|}{ Itérations } & \\
\hline Candidats & T1 & T2 & T3 & T4 & T5 & T6 & T7 & T8 & T9 & T10 & Class. \\
\hline Cheminade & 0 & & & & & & & & & & 11 \\
\hline Lassalle & 1,68 & 1,68 & & & & & & & & & 10 \\
\hline Asselineau & 4,32 & 4,32 & 4,32 & & & & & & & & 9 \\
\hline Arthaud & 9,48 & 9,48 & 9,48 & 9,48 & & & & & & & 8 \\
\hline Poutou & 10,8 & 10,8 & 10,8 & 11,52 & 12,55 & & & & & & 7 \\
\hline Dupont-A, & 13,35 & 13,35 & 13,35 & 14,07 & 15,10 & 16,08 & & & & & 6 \\
\hline Hamon & 44,17 & 44,17 & 44,17 & 45,61 & 46,41 & 47,75 & 50,76 & & & & 5 \\
\hline Le Pen & 48,42 & 48,42 & 48,42 & 49,14 & 49,14 & 49,86 & 53,16 & 55,55 & & & 4 \\
\hline Fillon & 72,18 & 72,18 & 72,18 & 72,18 & 72,18 & 72,18 & 77,04 & 78,40 & 95,76 & & 3 \\
\hline Macron & 110,11 & 110,11 & 111,79 & 111,79 & 112,82 & 113,31 & 119,31 & 136,74 & 161,91 & 187,61 & 2 \\
\hline Melenchon & 124,9 & 124,9 & 124,9 & 125,62 & 127,68 & 131,13 & 131,93 & 155,11 & 189,57 & 234,81 & 1 \\
\hline
\end{tabular}


On constate que le classement que l'on obtient diffère de quelque peu de celui que nous avons obtenu avec une correction au niveau national : J-L. Melenchon remporte maintenant le dernier duel face à E. Macron; F. Fillon passe de la quatrième à le troisième position tandis que M. Le Pen passe de troisième à quatrième. Les deux corrections s'accordent sur les autres positions.

\section{C.3. Les résultats de la règle de Borda à 4}

TABLE 14 - Borda à 4 avec correction niveau Martinique

\begin{tabular}{|c|c|c|c|c|c|c|c|c|c|c|c|}
\hline & \multicolumn{11}{|c|}{ Candidats } \\
\hline & $\begin{array}{l}\dot{<} \\
1 \\
\dot{1} \\
0 \\
0 \\
0 \\
0\end{array}$ & $\begin{array}{l}\tilde{\Xi} \\
\stackrel{0}{1} \\
\stackrel{\Xi}{\Xi}\end{array}$ & 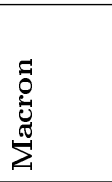 & 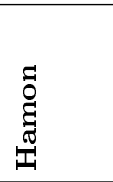 & 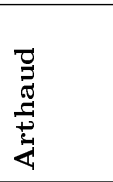 & $\begin{array}{l}\overrightarrow{0} \\
\stackrel{0}{0} \\
0 \\
0\end{array}$ & 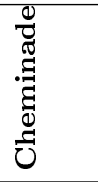 & 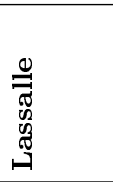 & 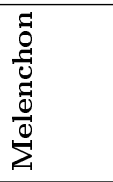 & 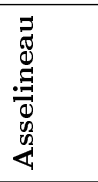 & $\stackrel{\text { }}{\stackrel{F}{F}}$ \\
\hline Scores corrigés & 184,10 & 404,50 & 873,29 & 678,85 & 197,60 & 268,13 & 73,50 & 109,63 & 926,56 & 97,12 & 509,02 \\
\hline Class. corrigé & 8 & 5 & 2 & 3 & 7 & 6 & 11 & 10 & 1 & 9 & 4 \\
\hline
\end{tabular}

On constate que lorsqu'on fait une correction avec les résultats de la Martinique, J-L. Mélenchon est le vainqueur de la règle de Borda à 4 ; aussi on constate que le classement collectif se rapproche de près de celui des résultats bruts. 


\section{Questionnaire}

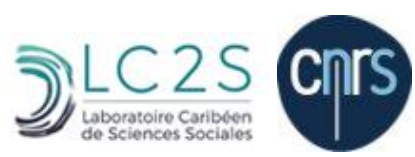

Vous pouvez nous aider dans notre expérience en consacrant quelques minutes de plus à cette étude. Nous vous en remercions par avance.

\section{Les modes de scrutin expérimentés}

1-Etes-vous satisfait de la règle utilisée actuellement pour l'élection présidentielle ?

0 - $\square$ Sans réponse 1- $\square$ Oui 2- $\square$ Non

L'élection officielle
\begin{tabular}{|l|l|l|}
\hline 2-J'ai voté pour : & 5- $\square$ M. Le Pen & 9- $\square$ E. Macron \\
\hline 1- $\square$ N. Dupont-Aignan & 6- $\square$ N. Arthaud & 10- $\square$ P. Poutou \\
\hline 2- $\square$ B. Hamon & 7- $\square$ J. Lassalle & 11- $\square$ JL. Melenchon \\
\hline 3- $\square$ J. Cheminade & 8- $\square$ F. Fillon & \\
\hline 4- $\square$ F. Asselineau &
\end{tabular}

12- $\square$ J'ai voté blanc

3-Qui êtes-vous?

1- $\square$ Femme 2- $\square$ Homme 3- $\square$ Sans réponse

4-Votre âge :

1- $\square$ 18-29 2- $\quad$ 30-39 3- $\square$ 40-49 4- $\square$ 50-59 5- $\square$ 60-69 6- $\square$ Plus de 70 7- $\square$ sans réponse

5-Votre catégorie professionnelle

1- $\square$ Retraités 2- $\square$ Eléves/Etudiants 3- $\square$ chômeurs 4- $\square$ Astisans etc 5- $\square$ employés

6- $\square$ Cadres et professions intellectuelles supérieures 7- $\square$ professions intermédiaires 8- $\square$ Pas de réponses 9- $\square$ sans profession

6-Avez-vous suivi les débats des candidats : $1-\square$ Oui $\quad 2-\square$ Non $\quad 3-\square$ sans réponse 
Bulletin de vote expérimental $n^{\circ} 1$

Vote Alternatif

Instructions :

Inscrire 1 dans la case située en face du candidat que vous préférez; 2 pour votre second choix, ainsi de suite jusqu'à 11 pour le candidat que vous classez dernier. Si le candidat que vous avez classé en 1 est éliminé (avec le plus petit nombre de première place), votre vote sera transféré au suivant. On procède à nouveau à l'élimination d'un candidat jusqu'à ce qu'il ne reste plus que celui qui sera le vainqueur de l'élection.

\begin{tabular}{|l|l|}
\hline Candidats & Position \\
\hline Nicolas DUPONT-AIGNAN & \\
\hline Marine LE PEN & \\
\hline Emmanuel MACRON & \\
\hline Benoît HAMON & \\
\hline Nathalie ARTHAUD & \\
\hline Philippe POUTOU & \\
\hline Jacques CHEMINADE & \\
\hline Jean LASSALLE & \\
\hline Jean-Luc MELENCHON & \\
\hline François ASSELINEAU & \\
\hline François FILLON & \\
\hline
\end{tabular}

Bulletin de vote expérimental $n^{\circ} 2$

Règle de Borda à 4

Instructions :

Parmi les 11 candidats, vous ne classez que quatre candidats en mettant 1 devant votre premier choix, et ainsi de suite jusqu'à 4.

Avec la règle de Borda à 4 , un candidat classé premier reçoit 4 points, le second 3 points, etc... et les non classés 0.

Le candidat élu est celui qui comptabilise la somme de notes la plus élevée.

\begin{tabular}{|l|l|}
\hline Nicolas DUPONT-AIGNAN & \\
\hline Marine LE PEN & \\
\hline Emmanuel MACRON & \\
\hline Benoît HAMON & \\
\hline Nathalie ARTHAUD & \\
\hline Philippe POUTOU & \\
\hline Jacques CHEMINADE & \\
\hline Jean LASSALLE & \\
\hline Jean-Luc MELENCHON & \\
\hline François ASSELINEAU & \\
\hline François FILLON & \\
\hline
\end{tabular}

\section{7-Lequel des modes de scrutin vous semble le plus satisfaisant ?}

$\square$ 1.Actuel $\square 2$. Vote Alternatif $\square 3$. Borda à $4 \square 4$. Sans réponse 


\section{Références}

AlœS-Ferrer C., Granic DG. [2010], Approval voting in Germany : description of a field experiment, In Laslier JF., Sanver R. (dir.), Handbook of approval voting, Heidelberg, Springer-verlag, 397-411.

Arrow K. [1951], Social choice and individual values, New York, John Wiley.

Balinski M., Laraki R., Laslier J-F., Van Der Straeten K. [2003], Le vote par assentiment : une expérience, Cahiers du Laboratoire d'Econométrie de l'Ecole Polytechnique, no 2003-13.

Balinski M., Laraki R. [2011], Election by majority Judgement : experimental evidence, In Grofman B., Laurent A., Dolez B. (eds.), In situ and Laboratory Experiments on Electoral Law Reform : French Presidential Elections, Heidelberg, Springer, 13-54.

Baujard A., Igersheim H. [2007], Expérimentation du vote par note et du vote par approbation lors des élections présidentielles françaises du 22 avril 2007, Rapport au Centre d'Analyse Stratégique.

Baujard A., Igersheim H. [2009], Expérimentation du vote par note et du vote par approbation le 22 avril 2007. Premiers résultats, Revue économique, 60 (1), 189-202.

Baujard A., Igersheim H., Laslier J-F. [2016], La question du vote : Expérimentations en laboratoire et in situ, L'Actualité Economique, 92 (1-2), 151-189.

Baujard A., Igersheim H., Senné T. [2011], Political supply in the 2007 French Presidential election : An analysis based on experimental data, Annales d'économie et de statistiques, 101-102, 142-178.

Baujard A., Gavrel F., Laslier J-F., Igersheim H., Lebon I. [2013], Vote par approbation, vote par note: Une expérimentation lors des Elections Présidentielles du 22 Avril 2012, Revue Economique, 64(2), 345-356.

Baujard, A., Gavrel F., Laslier J-F., Igersheim H., Lebon I. [2014], Who's favored by evaluative voting? An experiment conducted during the 2012 French Presidential Election, Electoral Studies, 34, 131-145.

Baujard A., Gavrel F., Laslier J.-F., Igersheim H., Lebon I. [2018], How voters use grade scales in evaluative voting?, European Journal of Political Economy, 55, 14-28.

Blais A., Laslier J-F., Poinas F., Van Der Straeten K. [2015], Citizens' preferences about voting rules : self-interest, ideology, and sincerity, Public Choice, 164, 423-442.

de Borda J.-C [1781], Mémoire sur les élections au scrutin, Académie Royale des Sciences, Paris.

Braconnier C., Coulmont B., Dormagen J.Y. [2017], Toujours pas de chrysanthèmes pour les variables lourdes de la participation électorale. Chute de la participation et augmentation des inégalités électorales au printemps 2017, Revue française de science politique, 67(6), 1023-1040. 
Brian E. [2001], Le suffrage et les savants. Actes de la recherche en sciences sociales, 140, 31-33.

Condorcet N. [1785], Essai sur l'application de l'analyse á la probabilité des décisions rendues à la pluralité des voix. Académie Royale des Sciences, Paris.

Darmann A., Gudner J., Klamler C. [2017], Election outcomes under different ways to announce preferences : an analysis of the 2015 parliament election in the Austrian federal state of Styria, Public Choice, 173, 201-216

Dolez B., Laurent A. [2001], Nationalisation des comportement electoraux en France, In Perrineau P., Reynié D. (dir), Dictionnaire du vote, Paris, Presses Universitaires de France, 682-683.

Duverger M. [1950], L’influence des systèmes électoraux sur la vie politique, Armand Colin, Paris.

Farrell D. M., Mackerras M., Mcallister I. [1996], Designing electoral institutions : STV systems and their consequences, Political Studies, 44, 24-43.

Farrell D. M., Mackerras M., Mcallister I. [2000], Through a glass darkly : understanding the world of STV, In Shaun Bowler and Bernard Grofman (editors), Elections in Australia, Ireland, and Malta under the Single Transferable Vote : reflections on an embedded institution, University of Michigan Press, 17-36.

Farvaque E., Jayet H., Ragot L. [2009], Quel mode de scrutin pour quel vainqueur? Une expérience sur le vote préférentiel transférable, Revue d'économie politique, 119, 221-246.

Farvaque E., Jayet H., Ragot L. [2011], French Presidential election : A field experiment on the single transferable vote, In Grofman B., Laurent A., DoLez B. (dir.), In situ and Laboratory Experiments on Electoral Law Reform : French Presidential Elections, Heidelberg, Springer, 55-68.

Felsenthal D., Nurmi H. [2018], Voting procedures for electing a single candidate, Springer International Publishing.

Gallagher M., Mitchell P. [2005], The politics of electoral systems, Oxford, New York, Oxford University Press.

Garrigou A. [1988], Le secret de l'isoloir. Actes de la recherche en sciences sociales, 71-72, $22-45$.

Gaxie D. [1978], Le cens caché : inégalités culturelles et ségrégation politique, Seuil, Paris.

Gaxie D. [1990], Au-delà des apparences. Sur quelques problèmes de mesure des opinions, Actes de la recherche en sciences sociales, 81-82, 97-113.

Gibbard A. [1973], Manipulation of voting schemes : A General Result, Econometrica, 41, 587-601. 
Gombin J. [2015], Le changement dans la continuité géographies électorales du Front National depuis 1992, In Crepon (dir.) Les faux-semblants du Front national, Paris : Presses de Sciences Po, 395-416.

Grofman B., Feld S. L. [2004], If you like the alternative vote (a.k.a. the instant runoff), then you ought to know about the Coombs rule, Electoral Studies, 23, 641-659.

Hare T. [1859], The election of representatives, parliamentary and municipal, Longmans Green, London.

Huc A. [2019], FN du Nord contre FN du Sud : Analyse sociogéographique des électorats Le Pen en 2017, Revue française de science politique, 69(2), 7-31.

Igersheim H., Baujard A. [2019], Expérimentation et théorie du vote, L'Économie politique, $82(2), 101-112$.

Igersheim H., Baujard, A., Gavrel F., Laslier J-F., Lebon I. [2016], Individual Behavior under Evaluative Voting : A comparison between laboratory and In Situ experiments, In Voting experiments, Blais A., Laslier J-F., and Van der Straeten K. (Eds.), Part IV, 257-269, Springer.

Kabre A., Laslier J-F., Van Der Straeten K. [2012], A propos de la polarisation politique en Afrique : Pouvoir soutenir plusieurs candidats?, Document de travail.

Laslier J-F. [2019], Voter Autrement : Le recours à l'évaluation, Collection du Cepremap no 51, Editions Rue d'Ulm.

Laslier J-F. [2012], And the Loser Is....Plurality Voting, In Electoral Systems, Felsenthal D., Machover M. (Eds), Studies in Choice and Welfare, Springer, Berlin, Heidelberg, 327-351.

Laslier J-F., Van Der Straeten K. [2004], Election présidentielle : Une expérience pour un autre mode de scrutin, Revue française de science politique, 54, 99-130.

Laslier J-F., Van Der Straeten K. [2008], Approval voting in the french 2002 presidential election : A live experiment, Experimental economics, 11, 97-105.

Lepelley D., Merlin V. [1999], Analyses géométriques et probabilistes des règles de vote avec une application au scrutin majoritaire à deux tours, Revue économique, 50, 699-714.

Martin P. [1996], Modes de scrutin : Mythes et réalités. Commentaire, 73, 85-92.

Moualek J. [2017], Des voix (vraiment) pas comme les autres : Les usages pluriels du vote blanc et nul, Revue française de science politique, 67, 1153-1166.

Norris P. [1997], Choosing electoral systems : Proportional, majoritarian and mixed systems, International Political Science Review, 18(3), 297-312.

Nurmi H. [1999], Voting paradoxes and how to deal with them, Springer-Verlag. 
Ranger J. [1970], L'électorat communiste dans l'élection présidentielle de 1969, Revue française de science politique, 20, 282-311.

Reilly B., Maley M. [2000], The Single Transferable Vote and the Alternative Vote compared. In Shaun Bowler and Bernard Grofman (editors), Elections in Australia, Ireland, and Malta under the Single Transferable Vote : reflections on an embedded institution, University of Michigan Press, 37-58.

Saari D.G. [1994], Geometry of voting, Springer-Verlag.

Saari D.G. [2003], Chaotic elections! A Mathematician Looks at Voting, Americam Math Society, AMS.

Sato S. [2016], Informational requirement of social rules to avoid the Condorcet loser : A characterization of the plurality with a runoff, Mathematical Social Sciences, 79, 11-19.

Satterthwaite M.A. [1975], Strategy-proofness and Arrow's conditions : Existence and correspondence theorems for voting procedures and social welfare functions, Journal of Economic Theory, 10(2) : 187-217.

Van Der Straeten K., Laslier J-F., Blais A. [2013], Vote au pluriel : How people vote when offered to vote under different rules?, Political Science and Politics, 46(2), 324-328. 